\title{
POINTWISE ESTIMATES FOR FIRST PASSAGE TIMES OF PERPETUITY SEQUENCES
}

\author{
D. BURACZEWSKI, E. DAMEK, AND J. ZIENKIEWICZ
}

\begin{abstract}
We consider first passage times $\tau_{u}=\inf \left\{n: Y_{n}>u\right\}$ for the perpetuity sequence

$$
Y_{n}=B_{1}+A_{1} B_{2}+\cdots+\left(A_{1} \ldots A_{n-1}\right) B_{n},
$$

where $\left(A_{n}, B_{n}\right)$ are i.i.d. random variables with values in $\mathbb{R}^{+} \times \mathbb{R}$. Recently, a number of limit theorems related to $\tau_{u}$ were proved including the law of large numbers, the central limit theorem and large deviations theorems (see [2]). We obtain a precise asymptotics of the sequence $\mathbb{P}\left[\tau_{u}=\log u / \rho\right], \rho>0, u \rightarrow \infty$ which considerably improves the previous results of [2]. There, probabilities $\mathbb{P}\left[\tau_{u} \in I_{u}\right]$ were identified, for some large intervals $I_{u}$ around $k_{u}$, with lengths growing at least as $\log \log u$. Remarkable analogies and differences to random walks $[7,17]$ are discussed.
\end{abstract}

\section{INTRODUCTION}

1.1. The perpetuity sequence and first passage times. Let $\left(A_{n}, B_{n}\right)$ be i.i.d. (independent identically distributed) random variables with values in $\mathbb{R}^{+} \times \mathbb{R}$. We consider the perpetuity sequence

$$
Y_{n}=B_{1}+A_{1} B_{2}+\cdots+\left(A_{1} \cdots A_{n-1}\right) B_{n}, \quad n=1,2, \ldots
$$

If $\mathbb{E}[\log A]<0$ and $\mathbb{E}\left[\log ^{+}|B|\right]<\infty, Y_{n}$ converges a.s. to the random variable

$$
Y=\sum_{n=0}^{\infty} A_{1} \ldots A_{n} B_{n+1},
$$

that is the unique solution to the random difference equation

$$
Y \stackrel{d}{=} A Y+B, \quad Y \text { independent of }(A, B) .
$$

The perpetuity process $\left\{Y_{n}\right\}$ is frequently present in both applied and theoretical problems. On one hand, the perpetuity sequence plays an important role in analyzing the ARCH and GARCH financial time series models, see Engle [10] and Mikosch [19]. On the other, it is connected to the random walk in random environment [16], the weighted branching process and the branching random walk, see Guivarc'h [13], Liu [18] and Buraczewski [1]. We refer the reader to recent monographs $[5,14]$ for an overview on the subject.

2010 Mathematics Subject Classification. Primary 60H25; secondary 60F10, 60J10.

Key words and phrases. Random recurrence equations, stochastic fixed point equations, first passage times, large deviations, asymptotic behavior, ruin probabilities.

The research was supported by the NCN grant DEC-2012/05/B/ST1/00692. 
The main objective of this paper is to study the asymptotic behavior of the first passage time

$$
\tau_{u}:=\inf \left\{n: Y_{n}>u\right\} \quad \text { as } u \rightarrow \infty .
$$

This is a basic question motivated partly by similar problems considered for random walks $\log \Pi_{n}=\log \left(A_{1} \ldots A_{n}\right)$, see the work of Lalley [17]. Some partial results in this direction were proved by Nyrhinen [20, 21]. An essential progress has been recently achieved in [2] and the aim of the present paper is to pursue the investigation further.

The tail of $Y$ was analysed under the Cramér condition

$$
\mathbb{E}\left[A^{\alpha_{0}}\right]=1 \text { for some } \alpha_{0}>0 \text {. }
$$

Then Kesten [15] and Goldie [12] proved that ${ }^{1}$

$$
\mathbb{P}[Y>u] \sim c_{+} u^{-\alpha_{0}} \quad \text { as } u \rightarrow \infty,
$$

which entails

$$
\mathbb{P}\left[\tau_{u}<\infty\right] \sim c_{0} u^{-\alpha_{0}} \quad \text { as } u \rightarrow \infty .
$$

In [2] the authors proved the law of large numbers ([2], Lemma 2.1)

$$
\frac{\tau_{u}}{\log u} \mid \tau_{u}<\infty \stackrel{\mathbb{P}}{\longrightarrow} \frac{1}{\rho_{0}}
$$

and the central limit theorem ([2], Theorem 2.2)

$$
\frac{\tau_{u}-\log u / \rho_{0}}{\sigma_{0} \rho_{0}^{-3 / 2} \sqrt{\log u}} \mid \tau_{u}<\infty \stackrel{d}{\longrightarrow} N(0,1)
$$

where $\rho_{0}=\mathbb{E}\left[A^{\alpha_{0}} \log A\right], \sigma_{0}=\mathbb{E}\left[A^{\alpha_{0}}(\log A)^{2}\right]$, and $\stackrel{\mathbb{P}}{\longrightarrow}($ resp. $\stackrel{d}{\longrightarrow})$ denotes convergence in probability (resp. in ditribution).

They also considered large deviations and showed that for $\rho>\rho_{0}$ ([2], Theorem 2.1)

$$
\mathbb{P}\left(\frac{\tau_{u}}{\log u}<\frac{1}{\rho}\right) \sim \frac{c(\rho)}{\sqrt{\log u}} u^{-I(\rho)}
$$

for some rate function $I(\rho)$ that will be defined below (see (1.5)). Indeed, the result in [2] is more precise and describes the asymptotic of

$$
\mathbb{P}\left[\tau_{u} \in I_{u}\right]
$$

for intervals $I_{u}$ around $\log u / \rho$ of the length of the order $\log \log u$. This implies that $\log u$ is the correct scaling for perpetuities as it is for the random walks. More details will be given below.

In this paper we go a step further and, under some continuity assumption on $A$, we describe the pointwise behavior of $\tau_{u}$, that is the asymptotic of

$$
\mathbb{P}\left[\tau_{u}=\lfloor\log u / \rho\rfloor\right], \quad u \rightarrow \infty
$$

for any $\rho>0$.

The results we obtain are partly with analogy to random walks but partly they are completely different and reveal essential differences in behavior of perpetuities and the corresponding random walk, see Theorems 1.6 and 1.14.

\footnotetext{
${ }^{1}$ We write $f(u) \sim g(u)$ for two functions $f$ and $g$, if $f(u) / g(u) \rightarrow 1$ as $u \rightarrow \infty$.
} 
1.2. Main results. While studying perpetuities the main role is played by fluctuations of the random walk $\Pi_{n}=A_{1} \ldots A_{n}$; see [5, 12]. In this paper the contractive case $\mathbb{E} \log A<0$ is studied, which entails that $\Pi_{n}$ converges to 0 a.s. On the other hand our hypotheses imply that $\mathbb{P}(A>1)>0$, thus the process $\Pi_{n}$ may attain (with small probability) arbitrary large values.

Properties of both processes $\left\{\Pi_{n}\right\}$ and $\left\{Y_{n}\right\}$ are essentially determined by the moments generating functions

$$
\lambda(s)=\mathbb{E}\left[A^{s}\right], \quad \text { and } \quad \Lambda(s)=\log \lambda(s) .
$$

We denote $\alpha_{\infty}=\sup \{\alpha: \lambda(\alpha)<\infty\}$ and $\alpha_{\min }=\operatorname{argmin} \lambda(\alpha)$. Then both functions $\lambda$ and $\Lambda$ are smooth and convex on their domain $\left[0, \alpha_{\infty}\right)$.

Recall that the convex conjugate (or the Fenchel-Legendre transform) of $\Lambda$ is defined by

$$
\Lambda^{*}(x)=\sup _{s \in \mathbb{R}}\{s x-\Lambda(s)\}, \quad x \in \mathbb{R} .
$$

$\Lambda^{*}$ appears in studying large deviations problems for random walks. Its various properties can be found in Dembo, Zeitouni [9]. Given $\alpha$ and $\rho=\Lambda^{\prime}(\alpha)$ we consider $\bar{\alpha}=\Lambda^{*}(\rho) / \rho$. An easy calculation shows that

$$
\bar{\alpha}=\alpha-\frac{\Lambda(\alpha)}{\Lambda^{\prime}(\alpha)}
$$

The parameter $\bar{\alpha}$ arises in the classical large deviations theory for random walks. As we will see below, $\bar{\alpha}$ plays a crucial role in our results. This parameter has a geometric interpretation: the tangent line to $\Lambda$ at point $(\alpha, \Lambda(\alpha))$ intersects the $x$-axis at $(\bar{\alpha}, 0)$. See the figure below.

Our main result is the following

Theorem 1.6. Given $\rho>0$ suppose there exists $\alpha<\alpha_{\infty}$ such that $\rho=\Lambda^{\prime}(\alpha)$. Assume additionally

(1.7) $\mathbb{E} \log A<0$;

(1.8) $\mathbb{E} A^{\alpha+\varepsilon}<\infty$ and $\mathbb{E}|B|^{\alpha+\varepsilon}<\infty$ for some $\varepsilon>0$;

(1.9) either $\alpha_{\min } \leq 1$ or $\Lambda(1)<\Lambda(\alpha)$;

(1.10) there are $\left(a_{1}, b_{1}\right),\left(a_{2}, b_{2}\right) \in \operatorname{supp} \mu$ such that $a_{1}<1, a_{2}>1$ and $\frac{b_{2}}{1-a_{2}}<\frac{b_{1}}{1-a_{1}}$;

(1.11) the law of $A$ has density $f_{A}(a)$ satisfying $f_{A}(a) \leq C(1+a)^{-D}$ for some $D>1+\alpha$;

(1.12) $\mathbb{P}[A \in d a, B \in d b] \leq f_{A}(a) d a d \nu(b)$ for some probability measure $\nu$.

Then

$$
\mathbb{P}\left[\tau_{u}=\lfloor\log u / \rho\rfloor\right] \sim \frac{c(\alpha) \lambda(\alpha)^{-\Theta(u)}}{\sqrt{\log u}} u^{-\bar{\alpha}}, \quad \text { as } u \rightarrow \infty,
$$

for some strictly positive constant $C$ and $\Theta(u)=k_{u}-\left\lfloor k_{u}\right\rfloor$, where $k_{u}=\log u / \rho$.

The next result shows that assumption (1.9) in Theorem 1.6 is indispensable and if $\rho=\Lambda^{\prime}(\alpha)$ is close to 0 , the behavior of the passage time may be of different order. 
Theorem 1.14. Given $\rho>0$ suppose there exists $\alpha<\alpha_{\infty}$ such that $\rho=\Lambda^{\prime}(\alpha)$ and hypothesis (1.7), (1.8) are satisfied. Assume additionally

$$
\begin{aligned}
& \Lambda(\alpha)<\Lambda(1) ; \\
& B>0, \text { a.s.; }
\end{aligned}
$$

there are $0<b_{1}<b_{2}$, a measure $\nu$ and a non vanishing continuous function $g_{A}$ such that

$$
\mathbb{P}\left[A \in d a, B_{n} \in \mathbf{1}_{\left(b_{1}, b_{2}\right)}(b) d b\right] \geq g_{A}(a) d a d \nu(b) .
$$

Then there exists $\delta>0$ such that

$$
\mathbb{P}\left[\tau_{u}=\lfloor\log u / \rho\rfloor\right] \geq \frac{C}{\sqrt{\log u}} u^{-\bar{\alpha}} u^{\delta} .
$$

1.3. Some comments. Study of $\tau_{u}$ is partly motivated by the work of Lalley [17], who considered this problem for the negatively drifted random walk $S_{n}=\log \Pi_{n}$. Let $\tau_{u}^{\prime}=$ $\inf \left\{n: S_{n}>\log u\right\}$. Lalley proved the central limit theorem (as in (1.4)) and described large deviations: $\mathbb{P}\left(\tau_{u}^{\prime}<\log u / \rho\right)$ for $\rho>\rho_{0}$ and $\mathbb{P}\left(\tau_{u}^{\prime}>\log u / \rho\right)$ for $\rho<\rho_{0}$. Recently Buraczewski and Maślanka [7] essentially improved his results applying the techniques introduced in the present paper. Pointwise estimates of $\tau_{u}^{\prime}$ analogous to (1.13) were obtained under hypotheses (1.7) and (1.8) only.

The general shape of (1.17) matches with the previous results [2] but now $\tau_{u}$ is much better localized. Moreover, contrary to [2], Theorem 1.6 is valid for all $\rho>0$. But there is a price to pay: considerably stronger assumptions than those of [2]:

- Hypothesis (1.9) indicates the optimal set of indices. Indeed, if $\alpha_{\min } \leq 1$ then (1.13) holds for every $\rho>0$ and $\alpha>\alpha_{\min }$. If not, we require the condition (1.15), which is well justified by Theorem 1.14 that provides a class of appropriate counterexamples to $(1.13)$.

If $\alpha_{\min }>1$ then there is $1<\tilde{\alpha}<\alpha_{0}$ such that $\Lambda(1)=\Lambda(\tilde{\alpha}),(1.15)$ is satisfied for $\alpha>\tilde{\alpha}$, and we have (1.13) for $\rho=\Lambda^{\prime}(\alpha)>\Lambda^{\prime}(\tilde{\alpha})$. If $\rho=\Lambda^{\prime}(\alpha)<\Lambda^{\prime}(\tilde{\alpha})$ the asymptotic may be of different order as we can see in Theorem 1.14.

- Assumption (1.10) is needed to ensure that the processes $Y_{n}$ exceeds with positive probability the level $u$ for an arbitrary large $u$ and this is the weakest assumption implying that (see [5], Proposition 2.5.4). Then, as explained in [3], the constants $c_{0}$ in (1.3) and $c(\alpha)$ in (1.13) are strictly positive.

- Assumptions (1.11) and (1.12) are technical. The strategy of the proof requires that $\mu$ - the joint law of $(A, B)$ is dominated by a product law and that the distribution of $A$ has a density that decays properly at $+\infty$.

- The function $\Theta(u)$ in (1.13) is a correction term, which is needed because $\tau_{u}$ is discrete, whereas $k_{u}$ depends on $u$ in a continuous way. Nevertheless, to simplify the notation, we avoid writing the integer part and below $k_{u}$ always denotes an integer number.

- The assumption (1.17) on $g_{A}$ can be improved to: $g_{A} d a$ is a measure containing non-trivial absolutely continuous part. We comment more on it in Remark 6.7 at the end of Section 6.

To prove the main results we analyze path properties of perpetuity sequence $Y_{n}$. This method, although technically quite involved, is ultimately very rewarding and finally it 
provides a much deeper insight into the process. This strategy has been used by Enriquez et al. [11] and Collamore, Vidyashankar [8] to obtain an explicit formula for the limiting constant $c_{+}$in (1.2) and also by Buraczewski et al. [4] to prove large deviations results.

1.4. Acknowledgements. We thank the referees for useful remarks which helped us to improve the previous version of the paper.

\section{LARGE DEVIATIONS FOR RANDOM WALKS}

To analyze the behavior of the random walk $\left\{\Pi_{n}\right\}$, the following uniform large deviation theorem, due to Petrov [22], Theorem 2, will play a key role (see also the Bahadur-Rao theorem in [9]).

Lemma 2.1 (Petrov). Assume that the law of $\log A$ is nonlattice and that $c$ satisfies $\mathbb{E}[\log A]<c<s_{0}$, and suppose that $\delta(n)$ is an arbitrary function satisfying $\lim _{n \rightarrow \infty} \delta(n)=$ 0 . Then with $\alpha$ chosen such that $\Lambda^{\prime}(\alpha)=c$, we have that

$$
\begin{aligned}
\mathbb{P}\left\{\Pi_{n}>\right. & \left.e^{n\left(c+\gamma_{n}\right)}\right\} \\
& =\frac{1}{\alpha \sigma(\alpha) \sqrt{2 \pi n}} \exp \left\{-n\left(\alpha\left(c+\gamma_{n}\right)-\Lambda(\alpha)+\frac{\gamma_{n}^{2}}{2 \sigma^{2}(\alpha)}\left(1+O\left(\left|\gamma_{n}\right|\right)\right)\right\}(1+o(1))\right.
\end{aligned}
$$

as $n \rightarrow \infty$, uniformly with respect to $c$ and $\gamma_{n}$ in the range

$$
\mathbb{E}[\log A]+\epsilon \leq c \leq s_{0}-\epsilon \text { and }\left|\gamma_{n}\right| \leq \delta(n),
$$

where $\epsilon>0$.

Remark 2.3. In $(2.2)$, we may have that $s_{0}=\infty$ or $\mathbb{E}[\log A]=-\infty$. In these cases, the quantities $\infty-\epsilon$ or $-\infty-\epsilon$ should be interpreted as arbitrary positive, respectively negative, constants.

In fact we will also use some refinements of the last result

Lemma 2.4. Under the hypotheses of the previous theorem assume additionally that $\delta_{n}, j_{n}$ satisfy

$$
\max \left\{\sqrt{n}\left|\delta_{n}\right|, j_{n} / \sqrt{n}\right\} \leq \delta(n)
$$

Then

$$
\mathbb{P}\left[\Pi_{n-j_{n}} \geq t e^{n \delta_{n}}\right]=\frac{1}{\alpha \sigma(\alpha) \sqrt{2 \pi n}} \cdot t^{-\bar{\alpha}} e^{-\alpha n \delta_{n}} e^{-j_{n} \Lambda(\alpha)}\left(1+o_{1}(1)\right) \quad \text { as } n \rightarrow \infty
$$

uniform for all $\Lambda^{\prime}(\alpha)$ satisfying $\mathbb{E} \log A_{1}+c_{1} \leq \Lambda^{\prime}(\alpha) \leq c_{2}, c_{1}, c_{2}>0, t=\exp \left(n \Lambda^{\prime}(\alpha)\right)$ and for all $\delta_{n}, j_{n}$ satisfying $(2.5)$.

Proof. Let $\bar{n}=\left(n-j_{n}\right)$ and $\rho=\Lambda^{\prime}(\alpha)$. We write $t e^{n \delta_{n}}=e^{n \rho+n \delta_{n}}$ and $n \rho+n \delta_{n}=$ $\left(n-j_{n}\right) \rho+j_{n} \rho+n \delta_{n}=\bar{n} \rho+\bar{n}\left(\frac{j_{n} \rho}{\bar{n}}+\frac{n \delta_{n}}{\bar{n}}\right)$. Now we may apply Lemma 2.1 with $\delta_{n}^{\prime}=\frac{j_{n} \rho}{\bar{n}}+\frac{n \delta_{n}}{\bar{n}}$ playing the role of $\gamma_{n}$ and $\bar{n}$ playing the role of $n$. Clearly then

$$
\bar{n}\left(\frac{j_{n} \rho}{\bar{n}}+\frac{n \delta_{n}}{\bar{n}}\right)^{2}=O(\delta(n)) .
$$


Hence we may write

$$
\begin{aligned}
\mathbb{P}\left[A_{1} . . A_{n-j_{n}} \geq t e^{n \delta_{n}}\right] & =\frac{1}{\alpha \sigma(\alpha) \sqrt{2 \pi n}} \cdot e^{-\rho \bar{n} \bar{\alpha}} e^{-\alpha \bar{n} \delta_{n}^{\prime}}\left(1+o_{1}(1)\right) \\
& =\frac{1}{\alpha \sigma(\alpha) \sqrt{2 \pi n}} \cdot e^{-\rho n \bar{\alpha}} e^{-\alpha n \delta_{n}} e^{-j_{n} \Lambda(\alpha)}\left(1+o_{1}(1)\right)
\end{aligned}
$$

which proves the lemma.

\section{Lower estimates in TheOrem 1.6}

In this section we prove lower estimates. Our main result is the following.

Proposition 3.1. Assume (1.7), (1.8) and (1.10) are satisfied, then there is $\eta>0$ such that

$$
\mathbb{P}\left[\tau_{u}=k_{u}+1\right]=\mathbb{P}\left[M_{k_{u}}<u \text { and } Y_{k_{u}+1}>u\right] \geq \frac{\eta}{\sqrt{\log u}} u^{-\bar{\alpha}}
$$

for $k_{u}=\frac{\log u}{\Lambda^{\prime}(\alpha)}$ and sufficiently large $u$, where $M_{n}$ denotes the sequence of maxima of $Y_{n}$ :

$$
M_{n}=\max \left\{0, Y_{1}, \ldots, Y_{n}\right\} .
$$

The proof of this Proposition is based on two lemmas. First we consider the following perpetuity

$$
\bar{Y}_{n}=\sum_{j=1}^{n} \Pi_{j-1}\left|B_{j}\right|
$$

and we study the joint distribution of $\Pi_{n}$ and $\bar{Y}_{n}$ as $n \rightarrow \infty$. As we will see below we are able to control probability of the event

$$
\left\{c_{1} u<\Pi_{k_{u}-j}<c_{2} u, \bar{Y}_{k_{u}-j}<\gamma u\right\},
$$

for some constants $\gamma<1, c_{1}, c_{2}, j$. Next we can choose $\left(\left(A_{k_{u}-j+1}, B_{k_{u}-j+1}\right), \ldots,\left(A_{k_{u}+1}, B_{k_{u}+1}\right)\right)$ with positive probability (depending on $c_{1}, c_{2}, \gamma, j$, but not on $u$ ) such that the corresponding perpetuity exceeds the level $u$ exactly at time $k_{u}+1$. Observe that this part of the proof does not require our continuity hypotheses (1.11) and (1.12).

Lemma 3.2. Assume (1.7) and (1.8). For a fixed $r_{0}>1$, there is $s \geq 1$, such that for every $\gamma>0,1<r \leq r_{0}$ and $u \geq u(\gamma, r)$

$$
\mathbb{P}\left\{\gamma u<\Pi_{k_{u}} \leq \gamma r u, \quad \bar{Y}_{k_{u}} \leq \gamma s u\right\} \geq \frac{D(r) \gamma^{-\alpha}}{\sqrt{\log u}} u^{-\bar{\alpha}},
$$

where $k_{u}=\frac{\log u}{\Lambda^{\prime}(\alpha)}$ and the function $D(r)>0$ for $1<r \leq r_{0}$ is increasing.

Remark 3.4. For given $r_{0}$ and $s$ the value of $u(\gamma, r)$ is not uniformly bounded in $\gamma, r \rightarrow 0$. The property that $s$ can be chosen independently of $\gamma>0$ and $1<r \leq r_{0}$ is crucial for our proof of Proposition 3.1.

Proof. By Lemma 2.1, there exists a constant $D(r)$ such that

$$
\mathbb{P}\left\{\gamma u<\Pi_{k_{u}} \leq \gamma r u\right\}=\frac{D(r)}{\sqrt{\log u}} \gamma^{-\alpha} u^{-\bar{\alpha}}(1+o(1)) \quad \text { as } \quad u \rightarrow \infty
$$


Recall $u^{-\bar{\alpha}}=u^{-\alpha}\left(\mathbb{E} A^{\alpha}\right)^{k_{u}}$. Notice we have

$$
\begin{aligned}
\mathbb{P}\left\{\gamma u \leq \Pi_{k_{u}}\right. & \left.\leq \gamma r u, \quad \bar{Y}_{k_{u}} \leq \gamma s u\right\} \\
& =\mathbb{P}\left\{\gamma u \leq \Pi_{k_{u}} \leq \gamma r u\right\}-\mathbb{P}\left\{\gamma u \leq \Pi_{k_{u}} \leq \gamma r u, \quad \bar{Y}_{k_{u}}>\gamma s u\right\}
\end{aligned}
$$

We are going to estimate the second summand in the last expression and prove

$$
\mathbb{P}\left\{\gamma u \leq \Pi_{k_{u}} \leq \gamma r u, \quad \bar{Y}_{k_{u}}>\gamma s u\right\} \leq \frac{C \gamma^{-\alpha}}{s^{\varepsilon}} u^{-\bar{\alpha}} \cdot\left(\frac{D(r)}{\sqrt{\log u}}+\frac{1}{\log u}\right)
$$

for $s \geq 1$ and $u \geq u(\gamma, r, s)$. Then we take $s=1+(2 C)^{\frac{1}{\varepsilon}}$, increase $u(\gamma, r, s)$ if necessarily and conclude the Lemma.

Notice that to prove (3.6), without any loss of generality, we may assume that $\left|B_{k}\right|>1$ a.s. We write

$$
\mathbb{P}\left\{\gamma u \leq \Pi_{k_{u}} \leq \gamma r u, \bar{Y}_{k_{u}}>\gamma s u\right\} \leq \sum_{i \geq 0} \mathbb{P}\left\{\gamma u \leq \Pi_{k_{u}} \leq \gamma r u, \Pi_{k_{u}-i-1}\left|B_{k_{u}-i}\right|>\frac{\gamma s u}{2(i+1)^{2}}\right\}
$$

We take large $K$ and we divide the sum into two parts depending whether $i>K \log k_{u}$ or $i \leq K \log k_{u}$.

Case 1. Suppose that $i>K \log k_{u}$. We take $\beta<\alpha$ and define $\varepsilon=\alpha-\beta, \delta=$ $\lambda(\beta) / \lambda(\alpha)<1$. Moreover, let $\Pi_{i}^{\prime}=A_{k_{u}-i+1} \cdots A_{k_{u}}$. We write

$$
\begin{aligned}
\mathbb{P}\{\gamma u \leq & \left.\Pi_{k_{u}} \leq \gamma r u, \quad \Pi_{k_{u}-i-1}\left|B_{k_{u}-i}\right|>\frac{\gamma s u}{2 i^{2}}\right\} \\
& \leq \sum_{m \geq 0} \mathbb{P}\left\{\Pi_{k_{u}} \geq \gamma u, \quad \frac{\gamma s u e^{m}}{2 i^{2}} \leq \Pi_{k_{u}-i-1}\left|B_{k_{u}-i}\right|<\frac{\gamma s u e^{m+1}}{2 i^{2}}\right\} \\
& =\sum_{m \geq 0} \mathbb{P}\left\{\Pi_{k_{u}-i-1} A_{k_{u}-i} \Pi_{i}^{\prime} \geq \gamma u, \quad \frac{\gamma s u e^{m}}{2 i^{2}} \leq \Pi_{k_{u}-i-1}\left|B_{k_{u}-i}\right|<\frac{\gamma s u e^{m+1}}{2 i^{2}}\right\} \\
& \leq \sum_{m \geq 0} \int \mathbb{P}\left\{\Pi_{k_{u}-i-1} a \Pi_{i}^{\prime} \geq \gamma u, \quad \frac{\gamma s u e^{m}}{2 i^{2}} \leq \Pi_{k_{u}-i-1} b<\frac{\gamma s u e^{m+1}}{2 i^{2}}\right\} \mu(d a d|b|) \\
& \leq \sum_{m \geq 0} \int \mathbb{P}\left\{\Pi_{k_{u}-i-1} \geq \frac{\gamma s u e^{m}}{2 i^{2} b}\right\} \cdot \mathbb{P}\left\{\Pi_{i}^{\prime} \geq \frac{2 i^{2} b}{a s e^{m+1}}\right\} \mu(d a d|b|)
\end{aligned}
$$


Now we apply twice the Chebyshev inequality and estimate the last expression by

$$
\begin{aligned}
\sum_{m \geq 0} \int \mathbb{P}\left\{\Pi_{k_{u}-i-1} \geq\right. & \left.\frac{\gamma s u e^{m}}{2 i^{2} b}\right\} \cdot \mathbb{P}\left\{\Pi_{i} \geq \frac{2 i^{2} b}{a s e^{m+1}}\right\} \mu(d a d|b|) \\
& \leq \sum_{m \geq 0} \int \frac{\left(2 i^{2} b\right)^{\alpha}}{\left(\gamma s u e^{m}\right)^{\alpha}} \lambda(\alpha)^{k_{u}-i} \cdot \frac{\left(a s e^{m}\right)^{\beta}}{\left(2 i^{2} b\right)^{\beta}} \lambda(\beta)^{i} \mu(d a d|b|) \\
& \leq \frac{2^{\alpha-\beta}}{\gamma^{\alpha} s^{\varepsilon}} \frac{1}{u^{\bar{\alpha}}} i^{2 \varepsilon} \delta^{i} \sum_{m \geq 0} e^{-m \varepsilon} \int b^{\varepsilon} a^{\beta} \mu(d a d|\beta|) \\
& =\frac{C i^{2 \varepsilon} \delta^{i}}{\gamma^{\alpha} s^{\varepsilon}} u^{-\bar{\alpha}}
\end{aligned}
$$

with the constant $C$ depending only on $\alpha, \beta$ and $\mu$. Summing over $i$ we obtain

$$
\begin{aligned}
\sum_{i>K \log k_{u}} \mathbb{P}\left\{\gamma u \leq \Pi_{k_{u}} \leq \gamma r u,\right. & \left.\Pi_{k_{u}-i-1}\left|B_{k_{u}-i}\right|>\frac{\gamma s u}{2 i^{2}}\right\} \\
\leq & \sum_{i>K \log k_{u}} \frac{C i^{2 \varepsilon} \delta^{i}}{\gamma^{\alpha} s^{\varepsilon}} u^{-\bar{\alpha}} \leq \frac{C}{s^{\varepsilon} \gamma^{\alpha} \log u} u^{-\bar{\alpha}}
\end{aligned}
$$

provided $K$ is sufficiently large. Note that we can choose $K$ depending only on $\mu, \alpha$ and $\beta$.

Case 2. Now we assume that $i \leq K \log k_{u}$ and that $L$ is large enough and satisfy

$$
\begin{aligned}
-\alpha L+1<0 & \text { if } \Lambda(\alpha) \geq 0, \\
-\alpha L+1-\Lambda(\alpha) K<0 & \text { if } \Lambda(\alpha)<0 .
\end{aligned}
$$

Then

$$
\begin{aligned}
\mathbb{P}\left\{\gamma u \leq \Pi_{k_{u}} \leq\right. & \left.\gamma r u, \quad \Pi_{k_{u}-i-1}\left|B_{k_{u}-i}\right|>\frac{\gamma s u}{2(i+1)^{2}}\right\} \\
\leq & \mathbb{P}\left\{\Pi_{k_{u}-i-1}\left|B_{k_{u}-i}\right| \geq \gamma s u \cdot k_{u}^{L}\right\} \\
& +\mathbb{P}\left\{\gamma u \leq \Pi_{k_{u}} \leq \gamma r u, \frac{\gamma s u}{2(i+1)^{2}} \leq \Pi_{k_{u}-i-1}\left|B_{k_{u}-i}\right| \leq \gamma s u \cdot k_{u}^{L}\right\}
\end{aligned}
$$

The first term is asymptotically negligible, since by the Chebyshev inequality and (3.7) (then the term in the brackets below is uniformly bounded for $i \leq K \log k_{u}$ )

$$
\begin{aligned}
\mathbb{P}\left\{\Pi_{k_{u}-i-1}\left|B_{k_{u}-i}\right| \geq \gamma s u \cdot k_{u}^{L}\right\} & \leq \mathbb{P}\left\{\Pi_{k_{u}-i-1} \geq \gamma s u \cdot k_{u}^{L}\right\} \\
& \leq \frac{1}{\gamma^{\alpha} s^{\alpha} u^{\alpha} k_{u}^{\alpha L}} \lambda(\alpha)^{k_{u}-i-1} \mathbb{E}\left[|B|^{\alpha}\right] \\
& \leq \frac{C \gamma^{-\alpha} s^{-\alpha}}{\log u} u^{-\bar{\alpha}}\left(k_{u}^{-\alpha L+1} e^{-(i+1) \Lambda(\alpha)}\right) \\
& \leq \frac{C}{\gamma^{\alpha} s^{\alpha} \log u} u^{-\bar{\alpha}}
\end{aligned}
$$

We will use this estimate later on. 
Next to estimate the second term in (3.8) we write

$$
\begin{aligned}
\mathbb{P}\left\{\gamma u \leq \Pi_{k_{u}} \leq\right. & \left.\gamma r u, \quad \frac{\gamma s u}{2 i^{2}} \leq \Pi_{k_{u}-i-1}\left|B_{k_{u}-i}\right| \leq \gamma s u \cdot k_{u}^{L}\right\} \\
& =\mathbb{P}\left\{\gamma u \leq \Pi_{k_{u}-i-1} A_{k_{u}-i} \Pi_{i}^{\prime} \leq \gamma r u, \quad \frac{\gamma s u}{2 i^{2}} \leq \Pi_{k_{u}-i-1}\left|B_{k_{u}-i}\right| \leq \gamma s u \cdot k_{u}^{L}\right\} \\
& \leq \sum_{0 \leq m \leq \log \left(2 i^{2} k_{u}^{L}\right)} \int \mathbb{P}(U(a, b, m)) \mu(d a, d|b|)
\end{aligned}
$$

for

$$
U(a, b, m)=\left\{\gamma u \leq \Pi_{k_{u}-i-1} a \Pi_{i}^{\prime} \leq \gamma r u, \quad \frac{\gamma s u e^{m}}{2 i^{2}} \leq \Pi_{k_{u}-i-1} b \leq \frac{\gamma s u \cdot e^{m+1}}{2 i^{2}}\right\} .
$$

Now we dominate the sets $U(a, b, m)$ as follows

$$
\begin{aligned}
\mathbb{P}(U(a, b, m)) & \leq \mathbb{P}\left\{\gamma u \leq \Pi_{k_{u}-i-1} a \Pi_{i}^{\prime} \leq \gamma r u, \quad \frac{2 b i^{2}}{s a e^{m+1}} \leq \Pi_{i}^{\prime} \leq \frac{2 r b i^{2}}{s a e^{m}}\right\} \\
& \leq \int \mathbb{P}\left\{\frac{\gamma u}{a w} \leq \Pi_{k_{u}-i-1} \leq \frac{\gamma r u}{a w}\right\} \mathbb{P}\left\{\frac{2 b i^{2}}{s a e^{m+1}} \leq \Pi_{i}^{\prime} \leq \frac{2 r b i^{2}}{s a e^{m}}, \Pi_{i}^{\prime} \in(w, w+d w)\right\} \\
& \leq \sup _{\frac{2 b i^{2}}{s a e^{m+1}} \leq w \leq \frac{2 r b i^{2}}{s a e^{m}}} \mathbb{P}\left\{\frac{\gamma u}{a w} \leq \Pi_{k_{u}-i-1} \leq \frac{\gamma r u}{a w}\right\} \mathbb{P}\left\{\frac{2 b i^{2}}{s a e^{m+1}} \leq \Pi_{i}^{\prime} \leq \frac{2 r b i^{2}}{s a e^{m}}\right\}
\end{aligned}
$$

Since both $m$ and $i$ are bounded by a constant times $\log k_{u}$, we can apply Petrov's result 2.1 on the set

$$
\Theta=\left\{b \leq e^{\sqrt{k_{u}}}\right\}
$$

Case 2a. Applying Lemma 2.1 and Chebyshev inequality we have

$$
\begin{aligned}
\sum_{0 \leq m \leq \log \left(2 i^{2} k_{u}^{L}\right)} & \int \mathbf{1}_{\Theta}(b) \mathbb{P}(U(a, b, m)) \mu(d a, d|b|) \\
& \leq \sum_{0 \leq m \leq \log \left(2 i^{2} k_{u}^{L}\right)} \int \mathbf{1}_{\Theta}(b) \frac{C D(r)}{\sqrt{k_{u}-i-1}} \frac{\left(i^{2} b\right)^{\alpha}}{\left(\gamma s e^{m}\right)^{\alpha}} \frac{\lambda(\alpha)^{k_{u}-i}}{u^{\alpha}} \frac{\left(a s e^{m}\right)^{\beta}}{\left(b i^{2}\right)^{\beta}} \lambda(\beta)^{i} \mu(d a, d|b|) \\
& \leq \frac{C D(r)}{\gamma^{\alpha} s^{\varepsilon}} \frac{1}{\sqrt{k_{u}}} u^{-\bar{\alpha}} i^{2 \varepsilon} \delta^{i} \sum_{m \geq 0} e^{-m \varepsilon} \cdot \int b^{\varepsilon} a^{\beta} \mu(d a, d|b|) \\
& \leq \frac{C D(r)}{\gamma^{\alpha} s^{\varepsilon} \sqrt{k_{u}}} u^{-\bar{\alpha}}
\end{aligned}
$$

with the constant $C$ depending only on $\alpha, \beta$ and $\mu$. 
Case 2b. Applying twice the Chebyshev inequality we obtain

$$
\begin{aligned}
& \sum_{0 \leq m \leq \log \left(2 i^{2} k_{u}^{L}\right)} \int \mathbf{1}_{\Theta^{c}}(b) \mathbb{P}(U(a, b, m)) \mu(d a, d|b|) \\
& \leq \sum_{0 \leq m \leq \log \left(2 i^{2} k_{u}^{L}\right)} \int \mathbf{1}_{\Theta^{c}}(b) \frac{\left(2 i^{2} b\right)^{\alpha}}{\left(\gamma s e^{m}\right)^{\alpha}} \cdot \frac{\lambda(\alpha)^{k_{u}-i}}{u^{\alpha}} \cdot \frac{\left(a s e^{m}\right)^{\beta}}{\left(b i^{2}\right)^{\beta}} \cdot \lambda(\beta)^{i} \mu(d a, d|b|) \\
& \leq \frac{2^{\alpha}}{\gamma^{\alpha} s^{\varepsilon}} u^{-\bar{\alpha}} i^{2 \varepsilon} \delta^{i} \sum_{m \geq 0} e^{-m \varepsilon} \cdot \int \mathbf{1}_{\Theta^{c}}(b) b^{\varepsilon} a^{\beta} \mu(d a, d|b|) \\
& \leq \frac{C}{\gamma^{\alpha} s^{\varepsilon}} u^{-\bar{\alpha}} \mathbb{E}|B|^{\varepsilon} A^{\beta} \mathbf{1}_{\Theta^{c}}
\end{aligned}
$$

We estimate the integral by the Hölder inequality with $p_{1}=\frac{\alpha}{\beta}, p_{2}=\frac{\alpha}{\epsilon}, \frac{1}{p_{1}}+\frac{1}{p_{2}}=1$, applied to variables $A^{\beta}, B^{\epsilon} \mathbf{1}_{\Theta^{c}}$. We get

$$
\left.\left.\int \mathbf{1}_{\Theta^{c}}(b) b^{\varepsilon} a^{\beta} \mu(d a, d|b|) \leq \mathbb{E}\left[A^{\alpha}\right]^{\frac{\beta}{\alpha}} \mathbb{E}|B|^{\alpha} \mathbf{1}_{\Theta^{c}}\right]^{\frac{\epsilon}{\alpha}} \leq e^{-\frac{\delta \epsilon}{\alpha} \sqrt{k_{u}}} \mathbb{E}\left[A^{\alpha}\right]^{\frac{\beta}{\alpha}} \mathbb{E}|B|^{\alpha+\delta}\right]^{\frac{\epsilon}{\alpha}} \leq \frac{C}{k_{u}}
$$

The second inequality follows by Chebyshev inequality.

Finally, by (3.9) and above estimates we obtain the estimate in case 2

$$
\begin{aligned}
\sum_{i \leq K \log k_{u}} \mathbb{P}\{\gamma u \leq & \left.\Pi_{k_{u}} \leq \gamma r u, \Pi_{k_{u}-i-1}\left|B_{k_{u}-i}\right|>\frac{\gamma s u}{2(i+1)^{2}}\right\} \\
& \left.\leq \frac{C}{\gamma^{\alpha} \sqrt{\log u}} u^{-\bar{\alpha}} \sum_{i \leq K \log k_{u}}\left(\frac{s^{-\alpha} k_{u}^{-\alpha L+1} e^{-i \Lambda(\alpha)}}{\sqrt{\log u}}+2 D(r) s^{-\varepsilon} i^{2 \varepsilon} \delta^{i}\right)\right) \\
& \leq \frac{C}{\gamma^{\alpha} \log u} u^{-\bar{\alpha}} s^{-\alpha}+\frac{C D(r)}{\gamma^{\alpha} \sqrt{\log u}} u^{-\bar{\alpha}} s^{-\epsilon}
\end{aligned}
$$

for $s \geq 1$. Combining both cases we obtain (3.6) and the lemma follows.

Lemma 3.10. Suppose that (1.10) is satisfied. Then there is $n$ such that

$$
\mathbb{P}\left[\Pi_{n}>1 \text { and } Y_{n}>0 \text { and } Y_{k}<Y_{n} \text { for } k=1, \ldots, n-1\right]>0 .
$$

In particular

$$
\mathbb{P}\left[\Pi_{n}>1 \text { and } Y_{n}>0\right]>0
$$

for some $n$.

Proof. Here we use assumption (1.10). Of course the lemma holds for $n=1$ when $b_{2}>0$, hence we assume in the proof $b_{2}<0$. Then $b_{1}>0$. We fix parameters $\delta, N, M$ (their values will be specified below). Define

$$
U_{\delta}(a, b)=\left\{\left(a^{\prime}, b^{\prime}\right):\left|a^{\prime}-a\right| \leq \delta a \text { and }\left|b^{\prime}-b\right| \leq \delta|b|\right\}
$$

and let

$$
\begin{aligned}
U=\left\{\left\{\left(A_{k}, B_{k}\right)\right\}_{k=1}^{N+M}:\right. & \left(A_{k}, B_{k}\right) \in U_{\delta}\left(a_{2}, b_{2}\right) \text { for } k=1, \ldots, N \\
& \left.\left(A_{k}, B_{k}\right) \in U_{\delta}\left(a_{1}, b_{1}\right) \text { for } k=N+1, \ldots, N+M\right\}
\end{aligned}
$$


for $\delta<\min \left\{\left|b_{2}\right|, b_{1}\right\}$. By assumption (1.10) the probability of $U$. From now we consider the perpetuity

$$
Y_{j}=\sum_{i=1}^{j} A_{1} \cdots A_{i-1} B_{i}=\sum_{i=1}^{j} \Pi_{i-1} B_{i} .
$$

on the set $U$. We have

$$
\begin{aligned}
Y_{N+M} & =Y_{N}+\Pi_{N} \sum_{j=1}^{M} a_{1}^{j-1} b_{1} \\
& \geq \sum_{j=1}^{N}\left((1+\delta) a_{2}\right)^{j-1} b_{2}(1+\delta)+\left((1-\delta) a_{2}\right)^{N} \sum_{j=1}^{M}\left((1-\delta) a_{1}\right)^{j-1} b_{1}(1-\delta) \\
& =b_{2}(1+\delta) \frac{(1+\delta)^{N} a_{2}^{N}-1}{(1+\delta) a_{2}-1}+(1-\delta)^{N} a_{2}^{N} \frac{(1-\delta)^{M} a_{1}^{M}-1}{(1-\delta) a_{1}-1} b_{1}(1-\delta) .
\end{aligned}
$$

Denote the last expression by $f(\delta)$. We will find integers $N$ and $M$ such that $a_{2}^{N} a_{1}^{M}>1$ and $f(0)>0$. Then by continuity, there exists $\delta>0$ such that $f(\delta)>0$ and simultaneously $\Pi_{N+M}=A_{1} \ldots A_{M+N}>1$ for any $A_{1}, \ldots, A_{M+N}$ in $U$. We have

$$
f(0)=\frac{b_{1}}{1-a_{1}}\left(1-a_{1}^{M}\right) a_{2}^{N}+\frac{b_{2}}{1-a_{2}}\left(1-a_{2}^{N}\right)
$$

To prove that the last expression is strictly positive recall

$$
\frac{b_{1}}{1-a_{1}}>\frac{b_{2}}{1-a_{2}}>0
$$

Since this is strict inequality and $a_{1}<1$ we can take large $M$ such that

$$
\frac{b_{1}}{1-a_{1}}\left(1-a_{1}^{M}\right)>\frac{b_{2}}{1-a_{2}} \text {. }
$$

Now, for any $N \geq 1$ we have

$$
\frac{b_{1}}{1-a_{1}}\left(1-a_{1}^{M}\right) a_{2}^{N}>\frac{b_{2}}{1-a_{2}} a_{2}^{N}>\frac{b_{2}}{1-a_{2}}\left(a_{2}^{N}-1\right)
$$

and this imply $f(0)>0$. We take $N$ large enough to satisfy $a_{1}^{M} a_{2}^{N}>1$.

Notice that $Y_{j}<0$ for $j \leq N$, hence

$$
Y_{j}<Y_{N+M} \text { for } j=1, \ldots, N \text {. }
$$

Moreover since for $j>N$

$$
Y_{j+1}-Y_{j}=\Pi_{j} B_{j+1}>0
$$

the sequence increases for $j>N$ and attains its maximum for $j=N+M$. Therefore also

$$
Y_{j}<Y_{N+M} \text { for } j=N+1, \ldots, N+M-1 \text {. }
$$

Finally since

$$
\mathbb{P}\left[\Pi_{N+M}>1 \text { and } Y_{N+M}>0 \text { and } Y_{k}<Y_{N+M} \text { for } k=1, \ldots, N+M-1\right] \geq \mathbb{P}(U)>0
$$

we conclude the lemma for $n=N+M$. 
Proof of Proposition 3.1. We will first prove the result under an additional assumption that $\mathbb{P}[A>1, B>0]>0$. Fix a point $\left(C_{A}, C_{B}\right) \in \operatorname{supp} \mu$ such that $C_{A}>1$ and $C_{B}>0$. Define

$$
\theta=\left(1-\frac{3}{4} \varepsilon_{0}\right)\left(\frac{C_{B}}{C_{A}^{j}} \frac{C_{A}^{j}-1}{C_{A}-1}\right)^{-1},
$$

Notice that for all $0<\varepsilon_{0} \leq 1$ and $j \geq 2$ we have the inequalities $\theta_{1} \geq \theta \geq \theta_{0}>0$, where constants $\theta_{0}, \theta_{1}$ depends on $C_{A}, C_{B}$ only. Fix any $\varepsilon_{0} \leq \frac{C_{A}-1}{4 C_{A}} \leq 1$ satisfying

$$
\theta_{0} C_{B}>2 \varepsilon_{0}
$$

Let $s_{0}$ satisfy the conclusion of Lemma 3.2 for $r_{0}=4$. We put $C_{2}=s_{0} \theta_{1}$ and fix large enough $j$ such that

$$
\frac{C_{2}}{C_{A}^{j}}<\frac{\varepsilon_{0}}{2}
$$

For very small $0<\delta \leq \frac{1}{2}$ (that will be defined slightly later) consider the set

$$
\begin{aligned}
\Omega=\left\{\frac{\theta u(1-\delta)}{C_{A}^{j}}<\Pi_{k_{u}-j}<\frac{\theta u(1+\delta)}{C_{A}^{j}},\right. & \bar{Y}_{k_{u}-j}<\frac{C_{2} u}{C_{A}^{j}} \\
& \left.\left(A_{k}^{\prime}, B_{k}^{\prime}\right) \in U_{\delta}\left(C_{A}, C_{B}\right) \text { for } k=1, \ldots, j+1\right\}
\end{aligned}
$$

Notice that on the set $\Omega$ we have

$$
\begin{aligned}
\Pi_{k_{u}-j} Y_{j}^{\prime} & =\Pi_{k_{u}-j}\left(B_{1}^{\prime}+A_{1}^{\prime} B_{2}^{\prime}+\cdots+A_{1}^{\prime} \ldots A_{j-1}^{\prime} B_{j}^{\prime}\right) \\
& \leq \frac{\theta u(1+\delta)}{C_{A}^{j}} \cdot(1+\delta) C_{B} \cdot \frac{C_{A}^{j}(1+\delta)^{j}-1}{C_{A}(1+\delta)-1} \\
& =D_{1}(\delta) u \\
\Pi_{k_{u}-j} Y_{j}^{\prime} & \geq \frac{\theta u(1-\delta)}{C_{A}^{j}} \cdot(1-\delta) C_{B} \cdot \frac{C_{A}^{j}(1-\delta)^{j}-1}{C_{A}(1-\delta)-1} \\
& =D_{2}(\delta) u \\
\Pi_{k_{u}} B_{k_{u}+1} & \geq \frac{\theta u(1-\delta)}{C_{A}^{j}} \cdot C_{A}^{j}(1-\delta)^{j} \cdot C_{B}(1-\delta)=\theta C_{B}(1-\delta)^{j+2} \\
& =D_{3}(\delta) u .
\end{aligned}
$$

moreover, by direct computation

$$
\begin{aligned}
& D_{1}(0)=D_{2}(0)=\frac{\theta C_{B}}{C_{A}^{j}} \cdot \frac{C_{A}^{j}-1}{C_{A}-1}=1-\frac{3}{4} \varepsilon_{0}, \\
& D_{3}(0)=\theta C_{B} \geq \theta_{0} C_{B} \geq 2 \varepsilon_{0} .
\end{aligned}
$$

Therefore, by continuity of $D_{i}(\delta)$ one can choose $\delta \leq \frac{1}{2}$ such that

$$
D_{1}(\delta)<1-\frac{\varepsilon_{0}}{2}, \quad D_{2}(\delta)>1-\varepsilon_{0}, \quad D_{3}(\delta) \geq \frac{3}{2} \varepsilon_{0} .
$$


Hence we have

$$
\begin{aligned}
\Omega & \subset\left\{\Pi_{k_{u}-j} Y_{j-1}^{\prime} \in\left(\left(1-\varepsilon_{0}\right) u,\left(1-\varepsilon_{0} / 2\right) u\right), \bar{Y}_{k_{u}-j}<\frac{\varepsilon_{0}}{2} u, \Pi_{k_{u}} B_{k_{u}+1} \geq(3 / 2) \varepsilon_{0} u, M_{k_{u}}<u\right\} \\
& \subset\left\{Y_{k_{u}} \in\left(\left(1-3 \varepsilon_{0} / 2\right) u, u\right), \Pi_{k_{u}} B_{k_{u}+1} \geq 3 \varepsilon_{0} u / 2, M_{k_{u}}<u\right\} \\
& \subset\left\{M_{k_{u}}<u \text { and } Y_{k_{u}+1}>u\right\},
\end{aligned}
$$

On the other hand by Lemma 3.2 applied with

$$
\gamma=\frac{\theta(1-\delta)}{C_{A}^{j}}, \quad r=\frac{1+\delta}{1-\delta}, \quad r_{0}=4, \quad \text { and } \quad s=\frac{C_{2}}{\theta(1-\delta)} \geq s_{0}=\frac{C_{2}}{\theta_{1}}
$$

we obtain

$$
\begin{aligned}
\mathbb{P}(\Omega)=\mathbb{P}\left\{\frac{\theta u(1-\delta)}{C_{A}^{j}}<\right. & \left.\Pi_{k_{u}-j}<\frac{\theta u(1+\delta)}{C_{A}^{j}}, \bar{Y}_{k_{u}-j}<\frac{C_{2} u}{C_{A}^{j}}\right\} \\
& \cdot \mathbb{P}\left\{\left(A_{k}^{\prime}, B_{k}^{\prime}\right) \in U_{\delta}\left(C_{A}, C_{B}\right) \text { for } k=1, . ., j+1\right\} \geq \frac{\eta}{\sqrt{\log u}} u^{-\bar{\alpha}}
\end{aligned}
$$

for some very small constant $\eta$.

If $\mathbb{P}[A>1, B>0]=0$ we apply Lemma 3.10 and proceed as above. This time we fix a point $\left(C_{A}, C_{B}\right) \in \operatorname{supp} \mu^{* n}$ such that $C_{A}>1$ and $C_{B}>0$, but instead of choosing $\left(A_{k}^{\prime}, B_{k}^{\prime}\right)$ with the law $\mu$ close to $\left(C_{A}, C_{B}\right)$ one has to pick up $\left(\tilde{A}_{k}, \tilde{B}_{k}\right)$ with the law $\mu^{* n}$ (i.e. partial products and perpetuities). This means:

$$
\begin{gathered}
\tilde{A}_{1}=A_{1}^{\prime} \cdots A_{n}^{\prime}, \quad \tilde{B}_{1}=\sum_{i=1}^{n} A_{1}^{\prime} \cdots A_{i-1}^{\prime} B_{i}^{\prime} \\
\tilde{A}_{k}=A_{(k-1) n+1}^{\prime} \cdots A_{k n}^{\prime}, \quad \tilde{B}_{k}=\sum_{i=(k-1) n+1}^{k n} A_{(k-1) n+1}^{\prime} \cdots A_{i-1}^{\prime} B_{i}^{\prime}
\end{gathered}
$$

and $\left(\tilde{A}_{k}, \tilde{B}_{k}\right)$ are chosen accordingly. Exactly the same calculations as above give the result. The condition $\left\{Y_{k}<Y_{n}, k<n\right\}$ in (3.11) is needed, to ensure that the perpetuity will not exceed the level $u$ before time $k_{u}$. We omit the details.

\section{Upper estimates in Theorem 1.6}

In this section we prove the following result, which gives upper estimates in Theorem 1.6

Proposition 4.1. Assume that (1.7), (1.8), (1.9), (1.11) and (1.12) are satisfied. Then there exists $C$ such that for every $u \geq 2$ we have

$$
\mathbb{P}\left[M_{k_{u}}<u, Y_{k_{u}+1}>u\right] \leq \mathbb{P}\left[Y_{k_{u}}<u, Y_{k_{u}+1}>u\right] \leq \frac{C}{\sqrt{\log u}} u^{-\bar{\alpha}}
$$

Moreover there is $\sigma<1$ and $C>0$ such that for every $\varepsilon>0$ and $u \geq 2$ we have

$$
\mathbb{P}\left[\tau_{u}=k_{u}+1\right] \leq \frac{C\left(\varepsilon^{1-\sigma}+u^{-\xi^{\prime}}\right)}{\sqrt{\log u}} u^{-\bar{\alpha}} .
$$


As in the previous Section we consider here the joint law of $\left(Y_{k_{u}}, \Pi_{k_{u}} B_{k_{u}+1}\right)$ and our main effort is to estimate

$$
\mathbb{P}\left[Y_{k_{u}} \in((1-\varepsilon) u,(1-\varepsilon / 2) u), \Pi_{k_{u}} B_{k_{u}+1}>\varepsilon u\right] .
$$

First we need two technical lemmas.

Lemma 4.3. Assume that (1.7) and (1.8) are satisfied. For any fixed (small) $\delta>0$ there exist $C_{\delta}$ such that for every $n \in \mathbb{Z}, \epsilon>0$ and $u \geq 2$ we have

$$
\mathbb{P}\left[\Pi_{k_{u}-1} B_{k_{u}}>\frac{\varepsilon u}{e^{n}}\right] \leq \frac{C_{\delta}}{\varepsilon^{\alpha}} \frac{e^{\alpha n+\delta|n|}}{\sqrt{\log u}} u^{-\bar{\alpha}}
$$

Remark 4.5. The above formula is meaningful only if the right hand side is smaller than 1 but it is useful to write the estimate in the unified way.

Proof. We consider three cases making distinction depending on whether $n, \log \left|B_{k_{u}}\right|$ are bigger or smaller than $\sqrt{k_{u}}$.

Case 1. Let first $\delta n>\sqrt{k_{u}}$. Then by the Chebychev inequality with exponent $\alpha$ we have

$$
\begin{aligned}
\mathbb{P}\left[\Pi_{k_{u}-1} B_{k_{u}}>\frac{\varepsilon u}{e^{n}}\right] & \leq C e^{k_{u} \Lambda(\alpha)} \mathbb{E}\left|B_{k_{u}}\right|^{\alpha} \varepsilon^{-\alpha} u^{-\alpha} e^{\alpha n} \\
& \leq C \varepsilon^{-\alpha} e^{\alpha n+\delta|n|} e^{-\sqrt{k_{u}}} u^{-\bar{\alpha}}
\end{aligned}
$$

and (4.4) follows.

Case 2. If $\log \left|B_{k_{u}}\right|>\sqrt{k_{u}}$, we write

$$
\begin{aligned}
\mathbb{P}\left[\Pi_{k_{u}-1} B_{k_{u}}>\frac{\varepsilon u}{e^{n}}\right] & \leq \sum_{m \geq \sqrt{k_{u}}} \mathbb{P}\left[\Pi_{k_{u}-1}>\frac{\varepsilon u}{e^{n+m+1}}\right] \mathbb{P}\left[B_{k_{u}}>e^{m}\right] \\
& \leq C e^{k_{u} \Lambda(\alpha)} \varepsilon^{-\alpha} u^{-\alpha} e^{\alpha n} \mathbb{E}\left|B_{k_{u}}\right|^{\alpha} \sum_{m \geq \sqrt{k_{u}}} e^{-\delta m} \\
& \leq C \varepsilon^{-\alpha} e^{\alpha n} e^{-\delta \sqrt{k_{u}}} u^{-\bar{\alpha}}
\end{aligned}
$$

which gives (4.4).

Case 3. If $\log \left|B_{k_{u}}\right| \leq \sqrt{k_{u}}$ and $n \leq \sqrt{k_{u}}$, we use Petrov theorem and we obtain

$$
\mathbb{P}\left[\Pi_{k_{u}-1} B_{k_{u}}>\frac{\varepsilon u}{e^{n}}\right] \leq C \frac{u^{-\bar{\alpha}}}{\sqrt{\log u}} \varepsilon^{-\alpha} e^{\alpha n} \mathbb{E}\left|B_{k_{u}}\right|^{\alpha},
$$

which completes the proof.

Let $B_{n}^{+}=\max \left\{B_{n}, 0\right\}$ and $Y_{n}^{+}=\sum_{k=1}^{n} \Pi_{k-1} B_{k}^{+}$. Then of course $Y_{n}^{+} \geq Y_{n}$.

Lemma 4.7. Assume that (1.7), (1.8) and (1.9) are satisfied. For any fixed (small) $\delta>0$ there exist $C_{\delta}$ and $\theta<1$ such that for every $n \in \mathbb{Z}, \epsilon>0, c \geq 1$ and $u \geq 2$ we have

$$
\mathbb{P}\left[\Pi_{k_{u}-1} B_{k_{u}}>\frac{\varepsilon u}{e^{n}} \quad \text { and } Y_{k_{u}-1}^{+}>\frac{c u}{e^{n}}\right] \leq \frac{C_{\delta}}{\varepsilon^{\theta}} \frac{e^{\alpha n+\delta|n|}}{\sqrt{\log u}} u^{-\bar{\alpha}}
$$

Remark 4.8. The above formula is meaningful only if the right hand side is smaller than 1 but it is usuful to write the estimate in the unified way. The Lemma will be used with fixed $c$. The condition $c \geq 1$ can be, of course, replaced by $c \geq c_{0}>0$. 
Proof. Denote

$$
g(n, m)=\mathbb{P}\left[\Pi_{k_{u}-1} B_{k_{u}}>\frac{\varepsilon u}{e^{n}} \text { and } \frac{c u e^{m}}{e^{n}}<Y_{k_{u}-1}^{+} \leq \frac{c u e^{m+1}}{e^{n}}\right]
$$

It is sufficient to prove that for $m \geq 0, n \in \mathbb{Z}$ and $u \geq$ const

$$
g(n, m) \leq \frac{C e^{-\delta m}}{\varepsilon^{\theta}} \frac{e^{\alpha n+\delta|n|}}{\sqrt{\log u}} u^{-\bar{\alpha}}
$$

Indeed, then

$$
\begin{aligned}
\mathbb{P}\left[\Pi_{k_{u}-1} B_{k_{u}}>\frac{\varepsilon u}{e^{n}} \text { and } Y_{k_{u}-1}^{+}>\frac{c u}{e^{n}}\right] & =\sum_{m \geq 0} g(n, m) \\
& \leq \sum_{m \geq 0} \frac{C e^{-\delta m}}{\varepsilon^{\theta}} \frac{e^{\alpha n+\delta|n|}}{\sqrt{\log u}} u^{-\bar{\alpha}} \\
& \leq \frac{C}{\varepsilon^{\theta}} \frac{e^{\alpha n+\delta|n|}}{\sqrt{\log u}} u^{-\bar{\alpha}}
\end{aligned}
$$

To estimate $g(n, m)$, for $j \geq 1$ we define the set of indices

$$
W_{j}^{u}=\left\{1 \leq i<k_{u}: \frac{c u e^{m}}{e^{n} e^{j}} \leq \Pi_{i-1} B_{i}^{+} \leq \frac{c u e^{m}}{e^{n} e^{j-1}}\right\}
$$

On the set $\left\{\frac{c u e^{m}}{e^{n}} \leq Y_{k_{u}-1}^{+}\right\}$there is some $j>0$, such that the number of elements in the set $W_{j}^{u}$ must be greater then $\frac{e^{j}}{10 j^{2}}$. Indeed, assume that such a $j$ does not exists, i.e. for every $j>0, \# W_{j}^{u} \leq \frac{e^{j}}{10 j^{2}}$, then

$$
\begin{aligned}
Y_{k_{u}-1}^{+} & =\sum_{i<k_{u}} \Pi_{i-1} B_{i}^{+}=\sum_{j>0} \sum_{i \in W_{j}} \Pi_{i-1} B_{i}^{+} \\
& \leq \sum_{j} \frac{e^{j}}{10 j^{2}} \cdot \frac{c u e^{m}}{e^{n} e^{j-1}}<\frac{c u e^{m}}{e^{n}} \sum_{j} \frac{e}{10 j^{2}} \\
& <\frac{c u e^{m}}{e^{n}} .
\end{aligned}
$$

Let

$$
K^{u}=\left\{\left(j, m_{1}, m_{2}\right): j \geq 1,1 \leq m_{1}<k_{u}, m_{1}+\frac{e^{j}}{10 j^{2}}<m_{2}<k_{u}\right\}
$$

Then

$$
g(n, m) \leq \sum_{\left(j, m_{1}, m_{2}\right) \in K^{u}} \mathbb{P}\left[U\left(j, m_{1}, m_{2}\right)\right]
$$

for

$$
U\left(j, m_{1}, m_{2}\right)=\left\{\frac{c u e^{m}}{e^{n} e^{j}} \leq \Pi_{m_{i}-1} B_{m_{i}}^{+} \leq \frac{c u e^{m}}{e^{n} e^{j-1}}, i=1,2, \text { and } \Pi_{k_{u}-1} B_{k_{u}}>\frac{\varepsilon u}{e^{n}}\right\} .
$$


Below we use the convention $\Pi_{k}, \Pi_{n}^{\prime}, \Pi_{m}^{\prime \prime}$ to denote independent products of $A_{j}$ 's of length $k, n, m$, respectively. Then for any triple $\left(j, m_{1}, m_{2}\right) \in K^{u}$ we have

$$
\begin{aligned}
\mathbb{P}\left[U\left(j, m_{1}, m_{2}\right)\right] & \\
\leq & \int \mathbb{P}\left[\frac{c u e^{m}}{e^{n} e^{j}} \leq \Pi_{m_{1}-1} b_{1} \leq \frac{c u e^{m}}{e^{n} e^{j-1}}, \frac{c u e^{m}}{e^{n} e^{j}} \leq \Pi_{m_{1}-1} a_{1} \Pi_{m_{2}-m_{1}}^{\prime} b_{2} \leq \frac{c u e^{m}}{e^{n} e^{j-1}},\right. \\
& \left.\Pi_{m_{1}-1} a_{1} \Pi_{m_{2}-m_{1}}^{\prime} a_{2} \Pi_{k_{u}-m_{2}}^{\prime \prime} b>\frac{\varepsilon u}{e^{n}}\right] \mathbf{1}_{\left\{b_{1}>0, b_{2}>0, b>0\right\}} \mu\left(d a_{1}, d b_{1}\right) \mu\left(d a_{2}, d b_{2}\right) \mu(d a, d b) \\
\leq & \int \mathbb{P}\left[\Pi_{m_{1}-1} \geq \frac{c u e^{m}}{b_{1} e^{n} e^{j}}\right] \cdot \mathbb{P}\left[\Pi_{m_{2}-m_{1}}>\frac{b_{1}}{b_{2} a_{1} e}\right] \cdot \mathbb{P}\left[\Pi_{k_{u}-m_{2}}>\frac{\varepsilon e^{j-1} b_{2}}{c b a_{2} e^{m}}\right] \\
& \cdot \mathbf{1}_{\left\{b_{1}>0, b_{2}>0, b>0\right\}} \mu\left(d a_{1}, d b_{1}\right) \mu\left(d a_{2}, d b_{2}\right) \mu(d a, d b)
\end{aligned}
$$

Fix parameters: $\beta_{1}=\alpha-\varepsilon_{1}, \beta_{2}=\alpha-\varepsilon_{2}$ such that $\beta_{1}, \beta_{2}<1, \rho_{1}=\frac{\lambda\left(\beta_{1}\right)}{\lambda(\alpha)}<1, \rho_{2}=\frac{\lambda\left(\beta_{2}\right)}{\lambda(\alpha)}<1$ and $\rho_{1}>\rho_{2}$. Here we use (1.9). If $\alpha_{\min }<1, \Lambda^{\prime}(\alpha)>0$ then we take $\alpha_{\min }<\beta_{2}<\beta_{1}<$ $\min \{1, \alpha\}$. If $\alpha_{\min } \geq 1$ and $\lambda(1)<\lambda(\alpha)$ then there is $\tilde{\alpha}<1$ such that $\lambda(\tilde{\alpha})=\lambda(\alpha)$ and so we can take $\beta_{1}=\tilde{\alpha}+\varepsilon_{1}, \beta_{2}=\tilde{\alpha}+\varepsilon_{2}$. We apply the Chebyshev inequality with parameters $\alpha, \beta_{1}, \beta_{2}$ and so

$$
\begin{aligned}
& \mathbb{P}\left[U\left(j, m_{1}, m_{2}\right)\right] \\
& \leq \int \frac{\left(b_{1} e^{n} e^{j}\right)^{\alpha}}{c^{\alpha} u^{\alpha} e^{\alpha m}} \lambda(\alpha)^{m_{1}} \cdot \frac{\left(b_{2} a_{1} e\right)^{\beta_{1}}}{b_{1}^{\beta_{1}}} \lambda\left(\beta_{1}\right)^{m_{2}-m_{1}} \frac{\left(c b a_{2} e^{m}\right)^{\beta_{2}}}{\left(\varepsilon e^{j-1} b_{2}\right)^{\beta_{2}}} \lambda\left(\beta_{2}\right)^{k_{u}-m_{2}} \\
& \cdot \mathbf{1}_{\left\{b_{1}>0, b_{2}>0, b>0\right\}} \mu\left(d a_{1}, d b_{1}\right) \mu\left(d a_{2}, d b_{2}\right) \mu(d a, d b) \\
& \leq C \varepsilon^{-\beta_{2}} e^{\alpha n} e^{-\varepsilon_{2} m} u^{-\bar{\alpha}} e^{j \varepsilon_{2}} \rho_{1}^{m_{2}-m_{1}} \rho_{2}^{k_{u}-m_{2}} \\
& \cdot \int b_{1}^{\varepsilon_{1}} b_{2}^{\beta_{1}-\beta_{2}} a_{1}^{\beta_{1}} a_{2}^{\beta_{2}} b^{\beta_{2}} \mathbf{1}_{\left\{b_{1}>0, b_{2}>0, b>0\right\}} \mu\left(d a_{1}, d b_{1}\right) \mu\left(d a_{2}, d b_{2}\right) \mu(d a, d b) \\
& \leq C \varepsilon^{-\beta_{2}} e^{\alpha n} e^{-\varepsilon_{2} m} u^{-\bar{\alpha}} e^{j \varepsilon_{2}} \rho_{1}^{m_{2}-m_{1}} \rho_{2}^{k_{u}-m_{2}} \mathbb{E}\left[\left|B_{1}\right|^{\varepsilon_{1}} A_{1}^{\beta_{1}}\right] \mathbb{E}\left[\left|B_{2}\right|^{\beta_{1}-\beta_{2}} A_{2}^{\beta_{2}}\right] \mathbb{E}|B|^{\beta_{2}}
\end{aligned}
$$

The product of expectations is finite, because of the Hölder inequality and (1.8). Hence it is sufficient to estimate

$$
\varepsilon^{-\beta_{2}} e^{\alpha n} e^{-\varepsilon_{2} m} u^{-\bar{\alpha}} \sum_{\left(j, m_{1}, m_{2}\right) \in K^{u}} e^{j \varepsilon_{2}} \rho_{1}^{m_{2}-m_{1}} \rho_{2}^{k_{u}-m_{2}} .
$$

Notice that the sum (of geometric sequence) above is always dominated by its maximal term, that is by $C k_{u}^{2 \varepsilon_{2}}<C k_{u}$. Assume first that $n>\sqrt{k_{u}}$. Then combining (4.10) with the estimates above

$$
\begin{aligned}
g(n, m) & \leq \sum_{\left(j, m_{1}, m_{2}\right) \in K^{u}} \mathbb{P}\left[U\left(j, m_{1}, m_{2}\right)\right] \\
& \leq \varepsilon^{-\beta_{2}} e^{\alpha n} e^{-\varepsilon_{2} m} u^{-\bar{\alpha}} k_{u}^{2} \\
& \leq \varepsilon^{-\beta_{2}} e^{(\alpha+\delta) n} e^{-\delta \sqrt{k_{u}}} e^{-\varepsilon_{2} m} u^{-\bar{\alpha}} k_{u}^{2} \\
& \leq \varepsilon^{-\beta_{2}} e^{(\alpha+\delta) n} e^{-\varepsilon_{2} m_{o}}\left(\frac{u^{-\bar{\alpha}}}{\sqrt{\log u}}\right)
\end{aligned}
$$


For the rest we fix $C_{1}$, we assume that $n \leq \sqrt{k_{u}}$ and we consider 4 cases

$$
\begin{aligned}
K_{1}^{u} & =\left\{\left(j, m_{1}, m_{2}\right) \in K^{u}: e^{j / 2}>C_{1} \log k_{u}\right\}, \\
K_{2}^{u} & =\left\{\left(j, m_{1}, m_{2}\right) \in K^{u}: e^{j / 2} \leq C_{1} \log k_{u}, m_{2}<k_{u}-k_{u}^{\frac{1}{4}}\right\}, \\
K_{3}^{u} & =\left\{\left(j, m_{1}, m_{2}\right) \in K^{u}: e^{j / 2} \leq C_{1} \log k_{u}, m_{1}<k_{u}-2 k_{u}^{\frac{1}{4}}, m_{2} \geq k_{u}-k_{u}^{\frac{1}{4}}\right\}, \\
K_{4}^{u} & =\left\{\left(j, m_{1}, m_{2}\right) \in K^{u}: e^{j / 2} \leq C_{1} \log k_{u}, m_{1} \geq k_{u}-2 k_{u}^{\frac{1}{4}}\right\} .
\end{aligned}
$$

Case 1. In this case there is $C_{2}$ such that $m_{2}-m_{1}>\frac{e^{j}}{10 j^{2}} \geq 2 C_{2} \log k_{u}$. Hence

$$
\begin{aligned}
g(n, m) & \leq C \varepsilon^{-\beta_{2}} e^{\alpha n} e^{-\varepsilon_{2} m} u^{-\bar{\alpha}} \sum_{\left(j, m_{1}, m_{2}\right) \in K_{1}^{u}} e^{j \varepsilon_{2}} \rho_{1}^{m_{2}-m_{1}} \rho_{2}^{k_{u}-m_{2}} \\
& \leq C \varepsilon^{-\beta_{2}} e^{\alpha n} e^{-\varepsilon_{2} m} u^{-\bar{\alpha}} k_{u} \rho_{1}^{C_{2} \log k_{u}} \\
& =\varepsilon^{-\beta_{2}} e^{\alpha n} e^{-\varepsilon_{2} m} o\left(\frac{u^{-\bar{\alpha}}}{\sqrt{\log u}}\right)
\end{aligned}
$$

Case 2. For the sum over $K_{2}^{u}$ we write

$$
\begin{aligned}
g(n, m) & \leq C \varepsilon^{-\beta_{2}} e^{\alpha n} e^{-\varepsilon_{2} m} u^{-\bar{\alpha}} \sum_{\left(j, m_{1}, m_{2}\right) \in K_{2}^{u}} e^{j \varepsilon_{2}} \rho_{1}^{m_{2}-m_{1}} \rho_{2}^{k_{u}-m_{2}} \\
& \leq C \varepsilon^{-\beta_{2}} e^{\alpha n} e^{-\varepsilon_{2} m} u^{-\bar{\alpha}} k_{u}\left(\log k_{u}\right)^{2 \varepsilon_{2}} \rho_{2}^{k_{u}^{\frac{1}{4}}} \\
& =\varepsilon^{-\beta_{2}} e^{\alpha n} e^{-\varepsilon_{2} m} o\left(\frac{u^{-\bar{\alpha}}}{\sqrt{\log u}}\right)
\end{aligned}
$$

Case 3. Here $m_{2}-m_{1}>k_{u}^{\frac{1}{4}}$ and reasoning as above

$$
\begin{aligned}
g(n, m) & \leq C \varepsilon^{-\beta_{2}} e^{\alpha n} e^{-\varepsilon_{2} m} u^{-\bar{\alpha}} \sum_{\left(j, m_{1}, m_{2}\right) \in K_{3}^{u}} e^{j \varepsilon_{2}} \rho_{1}^{m_{2}-m_{1}} \rho_{2}^{k_{u}-m_{2}} \\
& \leq C \varepsilon^{-\beta_{2}} e^{\alpha n} e^{-\varepsilon_{2} m} u^{-\bar{\alpha}} k_{u}^{2}\left(\log k_{u}\right)^{2 \varepsilon_{2}} \rho_{1}^{k_{u}^{\frac{1}{4}}} \\
& =\varepsilon^{-\beta_{2}} e^{\alpha n} e^{-\varepsilon_{2} m} o\left(\frac{u^{-\bar{\alpha}}}{\sqrt{\log u}}\right)
\end{aligned}
$$

Case 4a. On the set $\Theta=\left\{b_{1} \leq e^{\sqrt{k_{u}}}\right\}$ and $|n-m| \leq \sqrt{k_{u}}$ we estimate in a slightly different way the first term, that is

$$
\mathbb{P}\left[\Pi_{m_{1}} \geq \frac{c u e^{m}}{b_{1} e^{n} e^{j}}\right]
$$

We use Lemma 2.1 which gives

$$
\mathbb{P}\left[\Pi_{m_{1}} \geq \frac{c u e^{m}}{b_{1} e^{n} e^{j}}\right] \leq \frac{C\left(b_{1} e^{n} e^{j}\right)^{\alpha}}{c^{\alpha} u^{\alpha} e^{m \alpha}} \cdot \frac{\lambda(\alpha)^{m_{1}}}{\sqrt{m_{1}}} .
$$


Then, if $|n-m| \leq 2 \sqrt{k_{u}}$

$$
\begin{aligned}
\mathbb{P}\left[\Pi_{k_{u}-1} B_{k_{u}}>\frac{\varepsilon u}{e^{n}} \text { and } \frac{c u e^{m}}{e^{n}}\right. & \left.<Y_{k_{u}-1}^{+}<\frac{c u e^{m+1}}{e^{n}}, B \in \Theta\right] \\
& \leq C \varepsilon^{-\beta_{2}} e^{\alpha n} \frac{u^{-\bar{\alpha}}}{\sqrt{\log u}} e^{-\varepsilon_{2} m} \sum_{\left(j, m_{1}, m_{2}\right) \in K_{4}^{u}} e^{j \varepsilon} \rho_{1}^{m_{2}-m_{1}} \rho_{2}^{k_{u}-m_{2}} .
\end{aligned}
$$

Since, by the definition of $K^{u}$ the last sum is bounded, we obtain the required estimates.

Case 4b. If $m>k_{u}^{\frac{1}{4}}$ we use (4.11) and obtain

$$
g(n, m) \leq C \varepsilon^{-\beta_{2}} e^{\alpha n} e^{-\varepsilon_{2} m / 2} o\left(\frac{u^{-\bar{\alpha}}}{\sqrt{\log u}}\right) .
$$

Case 4c. If $n \leq-\sqrt{k_{u}}$ and $m<k_{u}^{\frac{1}{4}}$, then for large $u, e^{m} \leq e^{\frac{\delta|n|}{2}}$ and hence

$$
g(n, m) \leq \mathbb{P}\left[\Pi_{k_{u}-1} B_{k_{u}}>\frac{\varepsilon u}{e^{n}}\right] \leq \frac{e^{-\varepsilon_{1} m} e^{\alpha n+\delta|n|}}{\varepsilon^{\theta}} \frac{u^{-\bar{\alpha}}}{\sqrt{\log u}} .
$$

Case 4d. On the set $b_{1} \in \Theta^{c}$ we can sharpen (4.11). We get an extra decay for corresponding expectation $\int \mathbf{1}_{\Theta^{c}}\left(b_{1}\right) b_{1}^{\varepsilon_{1}} a_{1}^{\beta_{1}} \mu\left(d a_{1}, d b_{1}\right)$.

We use the Hölder inequality with $\frac{1}{p_{1}}+\frac{1}{p_{2}}=1, p_{1}$ close to 1 . Then

$$
\int \mathbf{1}_{\Theta^{c}}(b) b_{1}^{\varepsilon_{1}} a_{1}^{\beta_{1}} \mu\left(d a_{1}, d b_{1}\right) \leq \mathbb{E}\left[A^{p_{1} \beta_{1}}|B|^{p_{1} \varepsilon_{1}}\right]^{\frac{1}{p_{1}}} \cdot \mathbb{P}\left[|B|>e^{\sqrt{k_{u}}}\right]^{\frac{1}{p_{2}}}
$$

and the first term is finite agian by the Hölder inequality. Moreover, by (1.8).

$$
\mathbb{P}\left[|B|>e^{\sqrt{k_{u}}}\right]^{\frac{1}{p_{2}}} \leq\left(\mathbb{E}|B|^{\alpha}\right) e^{-\sqrt{k_{u}} \alpha / p_{2}}
$$

and we deduce as above.

Combining all the cases we obtain (4.9) and complete the proof of the Lemma.

Proof of Proposition 4.1. We are going to show that for every $0<\varepsilon<1$

$$
\mathbb{P}\left[Y_{k_{u}} \in((1-\varepsilon) u,(1-\varepsilon / 2) u), Y_{k_{u}+1}>u\right] \leq \varepsilon^{1-\sigma} \frac{C u^{-\bar{\alpha}}}{\sqrt{\log u}} .
$$

(4.13) implies (4.2). Moreover, applying (4.13) to $\varepsilon=2^{-n}, n=0,1,2 \ldots$ and summing up over $n$ we obtain Proposition 4.1. Let $J_{\varepsilon}=(1-\varepsilon, 1-\varepsilon / 2) u$. We write $Y_{k_{u}}=B_{1}+A_{1} Y_{k_{u}-1}^{\prime}$ where $Y_{k_{u}-1}^{\prime}=B_{2}+A_{2} B_{3}+\cdots+A_{2} \ldots A_{k_{u}-1} B_{k_{u}}$. We will also use notation $\Pi_{k_{u}-1}^{\prime}=$ $A_{2} \ldots A_{k_{u}}$. We have

$$
\mathbb{P}\left[Y_{k_{u}} \in J_{\varepsilon}, \Pi_{k_{u}} B_{k_{u}+1}>\varepsilon u\right]=\mathbb{P}\left[B_{1}+A_{1} Y_{k_{u}-1}^{\prime} \in J_{\varepsilon}, \Pi_{k_{u}} B_{k_{u}+1}>\varepsilon u\right]
$$


and

$$
\begin{aligned}
& \mathbb{P}\left[B_{1}+A_{1} Y_{k_{u}-1}^{\prime} \in J_{\varepsilon}, \Pi_{k_{u}} B_{k_{u}+1}>\varepsilon u\right] \\
& \leq \sum_{n \in \mathbb{Z}} \mathbb{P}\left[e^{n} \leq A_{1}<e^{n+1}, B_{1}+A_{1} Y_{k_{u}-1}^{\prime} \in J_{\varepsilon} \text { and } \Pi_{k_{u}-1}^{\prime} B_{k_{u}+1}>\frac{\varepsilon u}{e^{n+1}}\right] \\
& \leq \sum_{n \in \mathbb{Z}} \int \mathbf{1}_{\left\{\frac{u}{3 e^{n+1}}<s<\frac{3 u}{\left.e^{n}\right\}}\right.} \mathbb{P}\left[e^{n} \leq A_{1}<e^{n+1}, A_{1} \in \frac{1}{s}\left(J_{\varepsilon}-B_{1}\right)\right] \\
& \quad \cdot \mathbb{P}\left[\Pi_{k_{u}-1}^{\prime} B_{k_{u}}>\frac{\varepsilon u}{e^{n+1}}, Y_{k_{u}-1}^{\prime} \in d s\right]
\end{aligned}
$$

Notice that for $s \in\left(\frac{u}{3 e^{n+1}}, \frac{3 u}{e^{n}}\right)$ the interval $\frac{1}{s}\left(J_{\varepsilon}-B_{1}\right)$ has length at most $\frac{3}{2} \varepsilon e^{n}$. Thus, by Lemma 4.7

$$
\begin{aligned}
\mathbb{P}\left[B_{1}+\right. & \left.A_{1} Y_{k_{u}-1}^{\prime} \in J_{\varepsilon}, \Pi_{k_{u}} B_{k_{u}+1}>\varepsilon u\right] \\
& \leq \sum_{n \in \mathbb{Z}} \int \mathbf{1}_{\left\{\frac{u}{3 e^{n+1}}<s<\frac{3 u}{e^{n}}\right\}} C \varepsilon e^{n} \cdot \min \left\{e^{-n}, 1\right\}^{D} \cdot \mathbb{P}\left[\Pi_{k_{u}-1} B_{k_{u}}>\frac{\varepsilon u}{e^{n+1}}, Y_{k_{u}-1} \in d s\right] \\
& \leq \sum_{n \in \mathbb{Z}} C \varepsilon e^{n} \cdot \min \left\{e^{-n}, 1\right\}^{D} \cdot \mathbb{P}\left[\Pi_{k_{u}-1} B_{k_{u}}>\frac{\varepsilon u}{e^{n+1}}, \frac{u}{3 e^{n+2}}<Y_{k_{u}-1}<\frac{3 u}{e^{n}}\right] \\
& \leq \sum_{n \in \mathbb{Z}} C \varepsilon e^{n} \cdot \min \left\{e^{-n}, 1\right\}^{D} \cdot \mathbb{P}\left[\Pi_{k_{u}-1} B_{k_{u}}>\frac{\varepsilon u}{e^{n+1}}, Y_{k_{u}-1}^{+}>\frac{u}{3 e^{n+2}}\right] \\
& \leq \sum_{n \in \mathbb{Z}} C \varepsilon e^{n} \cdot \min \left\{e^{-n}, 1\right\}^{D} \frac{1}{\varepsilon^{\theta}} \frac{u^{-\bar{\alpha}}}{\sqrt{\log u}} \cdot e^{\alpha n+\delta|n|} \leq C \varepsilon^{1-\theta} \cdot \frac{u^{-\bar{\alpha}}}{\sqrt{\log u}}
\end{aligned}
$$

and Proposition (4.13) follows.

Lemma 4.15. Assume that (1.7), (1.8), (1.9), (1.11) and (1.12) are satisfied. For any fixed (large) $L>0$ there exist $C$ such that for every (small) $\epsilon, \eta>0,0<j \leq L$ and $u \geq 2$ we have

$$
\mathbb{P}\left[\Pi_{k_{u}-1} B_{k_{u}}>\varepsilon u, \Pi_{k_{u}-L} Y_{j}^{\prime} \in u\left(1-\eta^{L}, 1+\eta^{L}\right)\right] \leq C \varepsilon^{-\alpha} \eta^{L} \cdot \frac{u^{-\bar{\alpha}}}{\sqrt{\log u}}
$$

Moreover, for every $\epsilon, \eta>0$ and $u \geq 2$

$$
\mathbb{P}\left[\Pi_{k_{u}-1} B_{k_{u}}>\varepsilon u, \Pi_{k_{u}-L} M_{L}^{\prime} \in u\left(1-\eta^{L}, 1+\eta^{L}\right)\right] \leq C L \varepsilon^{-\alpha} \eta^{L} \cdot \frac{u^{-\bar{\alpha}}}{\sqrt{\log u}}
$$

and

$$
\mathbb{P}\left\{\Pi_{k_{u}} B_{k_{u}+1}>\varepsilon_{0} u, \Pi_{k_{u}-L} Y_{L}^{\prime}+\Pi_{k_{u}} B_{k_{u}+1} \in\left(1-\eta^{L}, 1+\eta^{L}\right) u,\right\} \leq C \varepsilon^{-\alpha} \eta^{L} \cdot \frac{u^{-\bar{\alpha}}}{\sqrt{\log u}}
$$


Proof. To prove (4.16), we use the same argument as for the proof of Proposition 4.1. Let $J_{\eta^{L}}=u\left(1-\eta^{L}, 1+\eta^{L}\right)$. We have

$$
\begin{aligned}
\mathbb{P}\left[\Pi_{k_{u}-L} Y_{j}^{\prime}\right. & \left.\in J_{\eta^{L}}, \Pi_{k_{u}-1} B_{k_{u}}>\varepsilon u\right] \\
= & \mathbb{P}\left[A_{1} \Pi_{k_{u}-L-1}^{\prime} Y_{j}^{\prime} \in J_{\eta^{L}}, A_{1} \Pi_{k_{u}-2}^{\prime} B_{k_{u}}>\varepsilon u\right] \\
\leq & \sum_{n \in \mathbb{Z}} \mathbb{P}\left[e^{n} \leq A_{1}<e^{n+1}, A_{1} \Pi_{k_{u}-L-1}^{\prime} Y_{j}^{\prime} \in J_{\eta^{L}} \text { and } \Pi_{k_{u}-2}^{\prime} B_{k_{u}}>\frac{\varepsilon u}{e^{n+1}}\right] \\
\leq & \sum_{n \in \mathbb{Z}} \int \mathbf{1}_{\left\{\frac{u}{2 e^{n+1}}<s<\frac{2 u}{e^{n}}\right\}} \mathbb{P}\left[e^{n} \leq A_{1}<e^{n+1}, A_{1} \in \frac{1}{s} J_{\eta^{L}}\right] \\
& \quad \cdot \mathbb{P}\left[\Pi_{k_{u}-2}^{\prime} B_{k_{u}}>\frac{\varepsilon u}{e^{n+1}}, \Pi_{k_{u}-L-1}^{\prime} Y_{j}^{\prime} \in d s\right]
\end{aligned}
$$

For $s$ in the domain of the integral, the length of $\frac{1}{s} J_{\eta^{L}}$ is at most $C \eta^{L} e^{n}$. As before, $\mathbb{P}\left[e^{n} \leq A_{1}<e^{n+1}, A_{1} \in \frac{1}{s} J_{\eta^{L}}\right] \leq C \eta^{L} e^{n} \cdot \min \left\{e^{-n}, 1\right\}^{D}$. Hence the last quantity of (4.19) is dominated by

$$
\sum_{n \in \mathbb{Z}} C \eta^{L} e^{n} \cdot \min \left\{e^{-n}, 1\right\}^{D} \cdot \mathbb{P}\left[\Pi_{k_{u}-2}^{\prime} B_{k_{u}}>\frac{\varepsilon u}{e^{n+1}}\right] .
$$

Now applying Lemma 4.3 for a fixed $\delta$ we obtain

$$
\begin{aligned}
\mathbb{P}\left[\Pi_{k_{u}-L} Y_{j}^{\prime} \in J_{\eta^{L}}, \Pi_{k_{u}-1} B_{k_{u}}>\varepsilon u\right] & \leq \sum_{n \in \mathbb{Z}} C \eta^{L} e^{n} \cdot \min \left\{e^{-n}, 1\right\}^{D} e^{\alpha n+\delta|n|} \frac{u^{-\bar{\alpha}}}{\sqrt{\log u}} \\
& \leq C \eta^{L} \varepsilon^{-\alpha} \cdot \frac{u^{-\bar{\alpha}}}{\sqrt{\log u}}
\end{aligned}
$$

and (4.16) follows.

The estimate (4.17) follows immediately from (4.16) and the definition $M_{L}^{\prime}$. The proof of (4.18) is similar to (4.16).

\section{Asymptotics}

Proof of Theorem 1.6. Step 1. Fix $\varepsilon_{0}$ and take u such that $\varepsilon_{0}>u^{-\xi^{\prime}}$. Then by Proposition 4.1

$$
\begin{aligned}
\mathbb{P}\left[\tau=k_{u}+1\right]= & \mathbb{P}\left[M_{k_{u}} \leq u, M_{k_{u}+1}>u\right] \\
= & \mathbb{P}\left[M_{k_{u}} \leq u, Y_{k_{u}+1}>u\right] \\
= & \mathbb{P}\left[M_{k_{u}} \leq u, Y_{k_{u}} \in\left[\left(1-\varepsilon_{0}\right) u, u\right], Y_{k_{u}+1}>u\right] \\
& +\mathbb{P}\left[M_{k_{u}} \leq u, Y_{k_{u}}<\left(1-\varepsilon_{0}\right) u, Y_{k_{u}+1}>u\right] \\
= & O\left(\frac{\varepsilon_{0}^{1-\sigma}}{\sqrt{\log u}} u^{-\bar{\alpha}}\right)+\mathbb{P}\left[M_{k_{u}} \leq u, Y_{k_{u}}<\left(1-\varepsilon_{0}\right) u, Y_{k_{u}+1}>u\right] .
\end{aligned}
$$

Thus is is sufficient to prove that

$$
\lim _{u \rightarrow \infty} u^{\bar{\alpha}} \sqrt{\log u} \mathbb{P}\left[M_{k_{u}} \leq u, Y_{k_{u}}<\left(1-\varepsilon_{0}\right) u, Y_{k_{u}+1}>u\right] \text { exists }
$$


for some fixed arbitrary small $\varepsilon_{0}$. Indeed, having proved (5.2) we first let $u \rightarrow \infty$ and then $\varepsilon_{0} \rightarrow 0$.

Similar arguments as in the proof of Lemma 4.7 show that there are constants: large $L_{0}$ and $\eta, \eta_{1}<1$ and $C$ possibly depending on $\epsilon_{0}$ such that for any $L>L_{0}$ we have

$$
\mathbb{P}\left[M_{k_{u}-L} \geq \eta^{L} u, \Pi_{k_{u}} B_{k_{u}+1}>\varepsilon_{0} u\right] \leq \eta_{1}^{L} \frac{C}{\sqrt{\log u}} u^{-\bar{\alpha}}
$$

and

$$
\mathbb{P}\left[\left|Y_{k_{u}-L}\right| \geq \eta^{L} u, \Pi_{k_{u}} B_{k_{u}+1}>\varepsilon_{0} u\right] \leq \eta_{1}^{L} \frac{C}{\sqrt{\log u}} u^{-\bar{\alpha}}
$$

Therefore, choosing large (but fixed) $L$, to prove the main result, it is enough to show that

$$
\lim _{u \rightarrow \infty} u^{\bar{\alpha}} \sqrt{\log u} \mathbb{P}[\Omega] \text { exists, }
$$

where

$$
\Omega=\left\{M_{k_{u}-L}<\eta^{L} u,\left|Y_{k_{u}-L}\right|<\eta^{L} u, M_{k_{u}}<u, Y_{k_{u}}<\left(1-\varepsilon_{0}\right) u, Y_{k_{u}+1}>u\right\} .
$$

As before, we conclude letting first $u \rightarrow \infty$ then $L \rightarrow \infty$. The limit in (5.5), if it exists, has to be positive. Indeed, taking $L$ large, we can make the upper bounds in (5.3) and (5.4) smaller than the lower bound in Proposition 3.1.

Step 2. To prove (5.5) we modify further the set $\Omega$ and as we will see, it is sufficient to replace $\Omega$ by a set $\Omega_{2}$ defined below. By the definition of $M_{k_{u}}$

$$
M_{k_{u}}=\max _{j=1, \ldots, k_{u}}\left\{Y_{j}\right\}=\max \left\{M_{k_{u}-L}, Y_{k_{u}-L}+\Pi_{k_{u}-L} M_{L}^{\prime}\right\}
$$

where $M_{L}^{\prime}=\max _{j=1, \ldots, L} \sum_{i=k_{u}-L}^{j} A_{k_{n}-L+1} \cdots A_{i-1} B_{i}$. Notice that $M_{L}^{\prime}$ has the same law as $M_{L}$.

Define the sets

$$
\begin{aligned}
\Omega_{1}= & \left\{M_{k_{u}-L}<\eta^{L} u, \Pi_{k_{u}-L} M_{L}^{\prime}<\left(1-\eta^{L}\right) u,\left|Y_{k_{u}-L}\right|<\eta^{L} u, \Pi_{k_{u}-L} Y_{L}^{\prime}<\left(1-\varepsilon_{0}-\eta^{L}\right) u,\right. \\
& \left.\Pi_{k_{u}-L} Y_{L}^{\prime}+\Pi_{k_{u}} B_{k_{u}+1}>\left(1+\eta^{L}\right) u\right\} \\
\Omega_{2}= & \left\{\Pi_{k_{u}-L} M_{L}^{\prime}<\left(1+\eta^{L}\right) u, \Pi_{k_{u}-L} Y_{L}^{\prime}<\left(1-\varepsilon_{0}+\eta^{L}\right) u, \Pi_{k_{u}-L} Y_{L}^{\prime}+\Pi_{k_{u}} B_{k_{u}+1}>\left(1-\eta^{L}\right) u\right\}
\end{aligned}
$$

Then

$$
\Omega_{1} \subset \Omega \subset \Omega_{2} \text {. }
$$

It is convenient to modify slightly $\Omega_{1}$ and consider

$$
\begin{aligned}
\Omega_{1}^{\prime}= & \Omega_{1} \cup \Omega_{1}^{\prime \prime} \\
= & \left\{\Pi_{k_{u}-L} M_{L}^{\prime}<\left(1-\eta^{L}\right) u, \Pi_{k_{u}-L} Y_{L}^{\prime}<\left(1-\varepsilon_{0}-\eta^{L}\right) u,\right. \\
& \left.\Pi_{k_{u}-L} Y_{L}^{\prime}+\Pi_{k_{u}} B_{k_{u}+1}>\left(1+\eta^{L}\right) u\right\}
\end{aligned}
$$


for

$$
\begin{aligned}
\Omega_{1}^{\prime \prime}=\{ & M_{k_{u}-L} \geq \eta^{L} u, \Pi_{k_{u}-L} M_{L}^{\prime}<\left(1-\eta^{L}\right) u, \Pi_{k_{u}-L} Y_{L}^{\prime}<\left(1-\varepsilon_{0}-\eta^{L}\right) u, \\
& \left.\Pi_{k_{u}-L} Y_{L}^{\prime}+\Pi_{k_{u}} B_{k_{u}+1}>\left(1+\eta^{L}\right) u\right\} \cup\left\{\left|Y_{k_{u}-L}\right| \geq \eta^{L} u, \Pi_{k_{u}-L} M_{L}^{\prime}<\left(1-\eta^{L}\right) u,\right. \\
& \left.\Pi_{k_{u}-L} Y_{L}^{\prime}<\left(1-\varepsilon_{0}-\eta^{L}\right) u, \Pi_{k_{u}-L} Y_{L}^{\prime}+\Pi_{k_{u}} B_{k_{u}+1}>\left(1+\eta^{L}\right) u\right\}
\end{aligned}
$$

Notice that by (5.3) and (5.4)

$$
\begin{aligned}
\mathbb{P}\left[\Omega_{1}^{\prime \prime}\right] & \leq \mathbb{P}\left[M_{k_{u}-L}>\eta^{L} u, \Pi_{k_{u}} B_{k_{u}+1}>\varepsilon_{0} u\right]+\mathbb{P}\left[\left|Y_{k_{u}-L}\right|>\eta^{L} u, \Pi_{k_{u}} B_{k_{u}+1}>\varepsilon_{0} u\right] \\
& \leq \eta_{1}^{L} \frac{C_{\varepsilon_{0}}}{\sqrt{\log u}} u^{-\bar{\alpha}} .
\end{aligned}
$$

We have

$$
\mathbb{P}\left[\Omega_{1}^{\prime}\right]-\mathbb{P}\left[\Omega_{1}^{\prime \prime}\right] \leq \mathbb{P}[\Omega] \leq \mathbb{P}\left[\Omega_{2}\right]
$$

We claim that $\mathbb{P}\left[\Omega_{2} \backslash \Omega_{1}^{\prime}\right] \leq C \varepsilon_{0}^{-\alpha} \eta^{L} \cdot \frac{u^{-\bar{\alpha}}}{\sqrt{\log u}}$. We have

$$
\begin{aligned}
\mathbb{P}\left[\Omega_{2} \backslash \Omega_{1}^{\prime}\right] & \leq \mathbb{P}\left\{\Pi_{k_{u}-L} M_{L}^{\prime} \in\left(1-\eta^{L}, 1+\eta^{L}\right) u, \Pi_{k_{u}} B_{k_{u}+1}>\varepsilon_{0} u\right\} \\
& +\mathbb{P}\left\{\Pi_{k_{u}-L} Y_{L}^{\prime} \in\left(1-\varepsilon_{0}-\eta^{L}, 1-\varepsilon_{0}+\eta^{L}\right) u, \Pi_{k_{u}} B_{k_{u}+1}>\varepsilon_{0} u\right\} \\
& +\mathbb{P}\left\{\Pi_{k_{u}-L} Y_{L}^{\prime}+\Pi_{k_{u}} B_{k_{u}+1} \in\left(1-\eta^{L}, 1+\eta^{L}\right) u, \Pi_{k_{u}} B_{k_{u}+1}>\varepsilon_{0} u\right\} .
\end{aligned}
$$

and the claim follows from Lemma 4.15 applied to each summand. Now it suffices to prove that

$$
\lim _{u \rightarrow \infty} u^{\bar{\alpha}} \sqrt{\log u} \mathbb{P}\left[\Omega_{2}\right] \quad \text { exists. }
$$

Step 3. To proceed we write

$$
\begin{aligned}
& P(u, \varepsilon, \delta, \gamma) \\
& \quad=\mathbb{P}\left[\Pi_{k_{u}-L} M_{L}^{\prime}<(1+\varepsilon) u, \Pi_{k_{u}-L} Y_{L}^{\prime}<(1+\delta) u, \Pi_{k_{u}-L} Y_{L}^{\prime}+\Pi_{k_{u}} B_{k_{u}+1}>(1+\gamma) u\right],
\end{aligned}
$$

where $\varepsilon, \delta, \gamma$ may have arbitrary signs. Notice that

$$
\mathbb{P}\left(\Omega_{2}\right)=P\left(u, \eta^{L},-\varepsilon_{0}+\eta^{L},-\eta^{L}\right) .
$$

We are going to prove that

$$
P(u, \varepsilon, \delta, \gamma)=C_{L} \frac{u^{-\bar{\alpha}}}{\sqrt{\log u}}(1+o(1))
$$

for some constant $C_{L}$. In fact, it is sufficient to show that

$$
\widetilde{P}(u, \varepsilon, \delta, \gamma)=C_{L} \frac{u^{-\bar{\alpha}}}{\sqrt{\log u}}(1+o(1)),
$$


where

$$
\begin{aligned}
& \widetilde{P}(u, \varepsilon, \delta, \gamma)=\mathbb{P}\left[\Pi_{k_{u}-L} M_{L}^{\prime}<(1+\varepsilon) u, \Pi_{k_{u}-L} Y_{L}^{\prime}<(1+\delta) u,\right. \\
& \Pi_{k_{u}-L} Y_{L+1}^{\prime}=\Pi_{k_{u}-L} Y_{L}^{\prime}+\Pi_{k_{u}} B_{k_{u}+1}>(1+\gamma) u, \text { and } u e^{\left.-(\log u)^{\frac{1}{4}}<\Pi_{k_{u}-L}<u e^{(\log u)^{\frac{1}{4}}}\right] .}
\end{aligned}
$$

Indeed

$$
\begin{aligned}
P(u, \varepsilon, \delta, \gamma)-\widetilde{P}(u, \varepsilon, \delta, \gamma) \leq & \mathbb{P}\left[\Pi_{k_{u}-L}>u e^{(\log u)^{\frac{1}{4}}}\right] \\
& +\mathbb{P}\left[\Pi_{k_{u}-L}<u e^{-(\log u)^{\frac{1}{4}}} \text { and } \Pi_{k_{u}} B_{k_{u}+1}>(\gamma-\delta) u\right] .
\end{aligned}
$$

Applying the Chebychev inequality with $\alpha$ to the first term we have

$$
\mathbb{P}\left[\Pi_{k_{u}-L}>u e^{(\log u)^{\frac{1}{4}}}\right] \leq e^{-\alpha(\log u)^{\frac{1}{4}}} u^{-\alpha} \lambda(\alpha)^{k_{u}-L}=o\left(\frac{u^{-\bar{\alpha}}}{\sqrt{\log u}}\right) .
$$

For the second term we choose $\beta>\alpha$ and we write

$$
\begin{aligned}
& \mathbb{P}\left[\Pi_{k_{u}-L}<u e^{-(\log u)^{\frac{1}{4}}} \text { and } \Pi_{k_{u}} B_{k_{u}+1}>(\gamma-\delta) u\right] \\
& \quad \leq \sum_{m \geq 0} \mathbb{P}\left[u e^{-(\log u)^{\frac{1}{4}}} e^{-(m+1)} \leq \Pi_{k_{u}-L}<u e^{-(\log u)^{\frac{1}{4}}} e^{-m}\right] \cdot \mathbb{P}\left[\Pi_{L} B>(\gamma-\delta) e^{(\log u)^{\frac{1}{4}}} e^{m}\right] \\
& \quad \leq \sum_{m \geq 0} \frac{C e^{\alpha(\log u)^{\frac{1}{4}}} e^{\alpha m}}{u^{\alpha}} \lambda(\alpha)^{k_{u}-L} \frac{\lambda(\beta)^{L} \mathbb{E}|B|^{\beta}}{e^{\beta(\log u)^{\frac{1}{4}}} e^{\beta m}} \\
& \quad=o\left(\frac{u^{-\bar{\alpha}}}{\sqrt{\log u}}\right),
\end{aligned}
$$

applying Chebychev with $\alpha$ and $\beta$ respectively. The our proof is reduced to (5.7).

Step 4. Finally, notice that $\widetilde{P}(u, \varepsilon, \delta, \gamma)$ is the probability of a set on which

$$
u e^{-(\log u)^{\frac{1}{4}}}<\Pi_{k_{u}-L}<u e^{(\log u)^{\frac{1}{4}}}
$$

Therefore, we may apply the Petrov theorem and we have

$$
\begin{aligned}
\widetilde{P}(u, \varepsilon, \delta, \gamma) & \\
& =\int \mathbb{P}\left[u \max \left(\frac{(1+\gamma)}{s_{3}}, \mathrm{e}^{-(\log u)^{\frac{1}{4}}}\right)<\Pi_{k_{u}-L}<u \min \left(\frac{(1+\varepsilon)}{s_{1}}, \frac{(1+\delta)}{s_{2}}, \mathrm{e}^{(\log u)^{\frac{1}{4}}}\right)\right] \\
& =\mathbb{P}\left[M_{L}^{\prime} \in\left(s_{1}, s_{1}+d s_{1}\right), Y_{L}^{\prime} \in\left(s_{2}, s_{2}+d s_{2}\right), Y_{L+1}^{\prime} \in\left(s_{3}, s_{3}+d s_{3}\right)\right] \\
& =\frac{C u^{-\bar{\alpha}}}{\sqrt{\log u}}(1+o(1)) \mathbb{E}\left[\left(\left(\frac{Y_{L+1}^{\prime}}{1+\gamma}\right)^{\alpha}-\max \left(\left(\frac{M_{L}^{\prime}}{(1+\varepsilon)}\right)^{\alpha},\left(\frac{Y_{L}^{\prime}}{(1+\delta)}\right)^{\alpha}\right)\right)_{+}\right]
\end{aligned}
$$

The last integral is finite by moment assumption (1.8) and the conclusion follows. 


\section{Proof of Theorem 1.14}

First for $\beta<\alpha+\xi$ we will need the following statement which is, in fact, Lemma 3.2 with $r=D_{1}$.

Lemma 6.1. Under assumptions of Theorem 1.14, there are constants $D_{1}, C_{1}, C$ such that for $n=\frac{\log u}{\Lambda^{\prime}(\beta)}$ and every $\gamma>0$

$$
\mathbb{P}\left[\gamma u<\Pi_{n}<D_{1} \gamma u, Y_{n}<C_{1} \gamma u\right] \geq \frac{C \lambda(\beta)^{n}}{u^{\beta} \gamma^{\beta} \sqrt{\log u}}
$$

Lemma 6.2. Under assumptions of Theorem 1.14, there are constants $D_{2}, C_{2}, C_{3}, C, m_{0}$ be such that for any $m>m_{0}$ and $\varepsilon=e^{m \Lambda^{\prime}(1)}<1$

$$
\mathbb{P}\left[\varepsilon \leq \Pi_{m} \leq D_{2} \varepsilon, C_{3} \leq Y_{m} \leq C_{2}\right] \geq \frac{C \lambda(1)^{m}}{\varepsilon \sqrt{m}}
$$

Proof. Step 1. We change the probability space and consider the probability measure $\lambda(1)^{-1} a \mu(d a, d b)$. Denote by $\mathbb{P}_{1}$ the corresponding probability measure on the space of trajectories and by $\mathbb{E}_{1}$ the corresponding expected value. Then, for $S_{m}=\log \Pi_{m}$,

$$
\begin{aligned}
\mathbb{P}\left[\varepsilon \leq \Pi_{m} \leq D_{2} \varepsilon\right] & \geq \frac{\lambda(1)^{m}}{D_{2} \varepsilon} \mathbb{E}\left[\mathbf{1}_{\left\{\varepsilon \leq \Pi_{m} \leq D_{2} \varepsilon\right\}} \frac{\Pi_{m}}{\lambda(1)^{m}}\right] \\
& =\frac{\lambda(1)^{m}}{D_{2} \varepsilon} \mathbb{P}_{1}\left[0 \leq S_{m}-m \Lambda^{\prime}(1) \leq \log D_{2}\right]
\end{aligned}
$$

Since $S_{m}$ is a sum of iid random variables, $\mathbb{E}_{1} S_{m}=m \Lambda^{\prime}(1)$ and $\mathbb{E}_{1} S_{1}^{2}<\infty$ (because of (H2)), by the local limit theorem

$$
\mathbb{P}\left[\varepsilon \leq \Pi_{m} \leq D_{2} \varepsilon\right] \geq \frac{C \lambda(1)^{m}}{\varepsilon \sqrt{m}}
$$

Step 2. Denote $\Pi_{m-j-1}^{\prime}=A_{j+2} \ldots A_{m}$. We have

$$
\begin{aligned}
\mathbb{P}\left[\varepsilon \leq \Pi_{m} \leq D_{2} \varepsilon, Y_{m} \geq C_{2}\right] & \leq \sum_{j=1}^{m-1} \mathbb{P}\left[\varepsilon<\Pi_{m} \leq D_{2} \varepsilon, \Pi_{j} B_{j+1}>\frac{C_{2}}{2 j^{2}}\right] \\
& =\sum_{j=1}^{m-1} \int \mathbb{P}\left[\varepsilon<\Pi_{j} a \Pi_{m-j-1}^{\prime}<D_{2} \varepsilon, \Pi_{j} b>\frac{C_{2}}{2 j^{2}}\right] \mu(d a, d b)
\end{aligned}
$$

and for every $j$ we consider

$$
P_{j}=\int \mathbb{P}\left[\varepsilon<\Pi_{j} a \Pi_{m-j-1}^{\prime}<D_{2} \varepsilon, \Pi_{j} b>\frac{C_{2}}{2 j^{2}}\right] \mu(d a, d b) .
$$


Step 2a. Since $\alpha_{\min }>1, \Lambda(1), \Lambda^{\prime}(1)<0$ and so we can choose $r<1$ and $\beta$ such that $r \Lambda(\beta)<\Lambda(1)-\Lambda^{\prime}(1)$ and $\Lambda(\beta)<0$. Then, by the Chebyshev inequality,

$$
\begin{aligned}
\sum_{j>r m} P_{j} & \leq \sum_{j>r m} \int \mathbb{P}\left[\Pi_{j} b>\frac{C_{2}}{2 j^{2}}\right] \mu(d a, d b) \\
& \leq \sum_{j>r m} \frac{\left(2 j^{2}\right)^{\beta}}{C_{2}^{\beta}} \lambda(\beta)^{j} \mathbb{E} B^{\beta} \leq C e^{r m(\Lambda(\beta)+\delta)} \\
& =o\left(\frac{\lambda(1)^{m}}{\varepsilon \sqrt{m}}\right)
\end{aligned}
$$

for a positive $\delta$ such that $r(\Lambda(\beta)+\delta)<\Lambda(1)-\Lambda^{\prime}(1)$.

Step 2b. For $j \leq r m$ we write

$$
\begin{aligned}
\sum_{j \leq r m} P_{j} & \leq \sum_{j \leq r m} \sum_{k \geq 0} \int \mathbb{P}\left[\varepsilon<\Pi_{j} a \Pi_{m-j-1}^{\prime}<D_{2} \varepsilon, \frac{C_{2} e^{k}}{2 j^{2} b}<\Pi_{j}<\frac{C_{2} e^{k+1}}{2 j^{2} b}\right] \mu(d a, d b) \\
& \leq \sum_{j \leq r m} \sum_{k \geq 0} \int \mathbb{P}\left[\frac{2 \varepsilon j^{2} b}{C_{2} a e^{k+1}}<\Pi_{m-j-1}<\frac{2 D_{2} \varepsilon j^{2} b}{C_{2} a e^{k}}\right] \cdot \mathbb{P}\left[\Pi_{j}>\frac{C_{2} e^{k}}{2 j^{2} b}\right] \mu(d a, d b)
\end{aligned}
$$

To proceed further we recall the Berry-Essen theorem (see e.g. [23]) that for an i.i.d. sequence $\left\{X_{j}\right\}$ with variance $\sigma^{2}$ and finite third moment, gives

$$
\sup _{x}\left|\mathbb{P}\left[\frac{\sum_{j=1}^{m} X_{j}-m \mathbb{E} X_{1}}{\sigma \sqrt{m}}<x\right]-\Phi(x)\right| \leq \frac{\bar{C} \mathbb{E}\left|X_{1}-\mathbb{E} X_{1}\right|^{3}}{\sigma^{3}} \cdot \frac{1}{\sqrt{m}}=\frac{\gamma}{\sqrt{m}}
$$

where $\Phi$ denotes the normal distribution and $\bar{C}$ is a universal constant. Hence, changing again the probability space, we have

$$
\begin{aligned}
\mathbb{P}\left[\frac{2 \varepsilon j^{2} b}{C_{2} a e^{k+1}}<\right. & \left.\Pi_{m-j-1}<\frac{2 D_{2} \varepsilon j^{2} b}{C_{2} a e^{k}}\right] \\
\leq & \frac{\lambda(1)^{m-j-1} C_{2} a e^{k+1}}{2 \varepsilon j^{2} b} \mathbb{E}\left[\mathbf{1}_{\left\{\frac{2 \varepsilon j^{2} b}{C_{2} a e^{k+1}}<\Pi_{m-j-1}<\frac{2 D_{2} \varepsilon j^{2} b}{C_{2} a e^{k}}\right\}} \frac{\Pi_{m-j-1}}{\lambda(1)^{m-j-1}}\right] \\
= & \frac{\lambda(1)^{m-j-1} C_{2} a e^{k+1}}{2 \varepsilon j^{2} b} \mathbb{P}_{1}\left[m \Lambda^{\prime}(1)+\log \left(2 j^{2} / C_{2}\right)+\log (b / a)-(k+1)<S_{m-j-1}\right. \\
& \left.<m \Lambda^{\prime}(1)+\log \left(2 D_{2} j^{2} / C_{2}\right)+\log (b / a)-k\right] \\
= & \frac{\lambda(1)^{m-j-1} C_{2} a e^{k+1}}{2 \varepsilon j^{2} b} \mathbb{P}_{1}\left[\frac{(j+1) \Lambda^{\prime}(1)+\log \left(2 j^{2} / C_{2}\right)+\log (b / a)-(k+1)}{\sqrt{m-j-1}}\right. \\
& \left.<\frac{S_{m-j-1}-(m-j-1) \Lambda^{\prime}(1)}{\sqrt{m-j-1}}<\frac{(j+1) \Lambda^{\prime}(1)+\log \left(2 D_{2} j^{2} / C_{2}\right)+\log (b / a)-k}{\sqrt{m-j-1}}\right] \\
\leq & \frac{\gamma C_{2} \lambda(1)^{m-j-1} a e^{k+1}}{2 \varepsilon j^{2} b \sqrt{m-j-1}} .
\end{aligned}
$$


(6.3) has been used in the last inequality. For $\mathbb{P}\left[\Pi_{j}>\frac{C_{2} e^{k}}{2 j^{2} b}\right]$ we use the Chebychev inequality with $\alpha$ and so

$$
\begin{aligned}
\sum_{j \leq r m} P_{j} & \leq C \sum_{j \leq r m} \sum_{k \geq 0} \frac{C_{2} \lambda(1)^{m-j-1} e^{k}}{\varepsilon j^{2} \sqrt{m-j-1}} \frac{j^{2 \alpha} \lambda(\alpha)^{j}}{C_{2}^{\alpha} e^{\alpha k}} \int a b^{\alpha-1} \mu(d a, d b) \\
& \leq C C_{2}^{1-\alpha} \frac{\lambda(1)^{m}}{\varepsilon \sqrt{m}} \sum_{j \leq r m}\left(\frac{\lambda(\alpha)}{\lambda(1)}\right)^{j} j^{2 \alpha} \\
& \leq \frac{C}{C_{2}^{\alpha-1}} \frac{\lambda(1)^{m}}{\varepsilon \sqrt{m}}
\end{aligned}
$$

Step 3. Combining first two steps and taking large $C_{2}$ we obtain

$$
\mathbb{P}\left[\varepsilon \leq \Pi_{m} \leq D_{2} \varepsilon, Y_{m} \leq C_{2}\right] \geq \frac{C \lambda(1)^{m}}{\varepsilon \sqrt{m}} .
$$

Take parameters $a_{2}>1, b_{1}<b_{2}, \eta>0$ such that

$$
\mathbb{P}\left[A \in\left(1, a_{2}\right), B \in\left(b_{1}, b_{2}\right)\right] \geq \eta>0
$$

Then, for $\left(A_{0}, B_{0}\right)$ independent of $\Pi_{m}$ and $Y_{m}$, we have

$$
\begin{aligned}
\eta \frac{C \lambda(1)^{m}}{\varepsilon \sqrt{m}} & \leq \mathbb{P}\left[A_{0} \in\left(1, a_{2}\right), B_{0} \in\left(b_{1}, b_{2}\right)\right] \cdot \mathbb{P}\left[\varepsilon \leq \Pi_{m} \leq D_{2} \varepsilon, Y_{m} \leq C_{2}\right] \\
& =\mathbb{P}\left[A_{0} \in\left(1, a_{2}\right), B_{0} \in\left(b_{1}, b_{2}\right) \text { and } \varepsilon \leq \Pi_{m} \leq D_{2} \varepsilon, Y_{m} \leq C_{2}\right] \\
& \leq \mathbb{P}\left[\varepsilon \leq \Pi_{m+1} \leq a_{2} D_{2} \varepsilon, b_{1} \leq Y_{m} \leq b_{2}+a_{2} C_{2}\right] .
\end{aligned}
$$

Thus the Lemma follows for $C_{3}=b_{1}$ and for $D_{2}, C_{2}$ replaced by $a_{2} D_{2}, b_{2}+a_{2} C_{2}$.

Proof of Theorem 1.14. Let $\beta>\alpha$ be very close to $\alpha$ and define $p$ by the relation

$$
\Lambda^{\prime}(\beta)=\frac{\Lambda^{\prime}(\alpha)}{p} \text {. }
$$

Since $\Lambda^{\prime \prime}(\beta)>0$, we have $p<1$. The precise value of $\beta$ will be chosen later on. Take $n=p k_{u}=\frac{\log u}{\Lambda^{\prime}(\beta)}$. Let $q=1-p, m=q k_{u}=k_{u}-n$. We write

$$
Y_{k_{u}}=Y_{n}+\Pi_{n-1} A_{n} Y_{m}^{\prime}=Y_{n-1}+\Pi_{n-1} B_{n}+\Pi_{n-1} A_{n} Y_{m}^{\prime}
$$

for $Y_{m}^{\prime}=\sum_{i=n+1}^{k_{u}} A_{n+1} \ldots A_{i-1} B_{i}$. Then $Y_{m}^{\prime}$ has the same law as $Y_{m}$. We denote also $\Pi_{j}^{\prime}=A_{n+1} \ldots A_{n+j}, B_{m}^{\prime}=B_{k_{u}}$ and we consider the set

$$
\Omega=\left\{\gamma u<\Pi_{n-1}<D_{1} \gamma u, Y_{n-1} \leq C_{1} \gamma u, C_{3} \leq Y_{m}^{\prime} \leq C_{2}, \varepsilon<\Pi_{m-1}^{\prime}<D_{2} \varepsilon, b_{1}<B_{m}^{\prime}<b_{2}\right\},
$$

where $\gamma, D_{1}$, are parameters given in Lemma $6.1, C_{2}, C_{3}, D_{2}$ are described in Lemma 6.2 $\varepsilon=e^{m \Lambda^{\prime}(1)}, b_{1}$ and $b_{2}$ are chosen as in (1.17).

By Lemmas 6.1 and 6.2

$$
\mathbb{P}(\Omega) \geq \frac{C \lambda(\beta)^{n}}{u^{\beta}} \frac{1}{\sqrt{n}} \frac{\lambda(1)^{m}}{\varepsilon \sqrt{m}}
$$


We write

$$
\begin{aligned}
\mathbb{P} & {\left[Y_{k_{u}-1} \leq u, Y_{k_{u}}>u\right] } \\
& =\mathbb{P}\left[Y_{n-1}+\Pi_{n-1} B_{n}+\Pi_{n-1} A_{n} Y_{m}^{\prime}-\Pi_{n-1} A_{n} \Pi_{m-1}^{\prime} B_{m} \leq u,\right. \\
& \left.Y_{n-1}+\Pi_{n-1} B_{n}+\Pi_{n-1} A_{n} Y_{m}^{\prime}>u\right] \\
& \geq \mathbb{P}\left[\left\{\frac{u-Y_{n-1}-\Pi_{n-1} B_{n}}{\Pi_{n-1} Y_{m}^{\prime}}<A_{n} \leq \frac{u-Y_{n-1}-\Pi_{n-1} B_{n}}{\Pi_{n-1}\left(Y_{m}^{\prime}-\Pi_{m-1}^{\prime} B_{m}^{\prime}\right)}, b_{1} \leq B_{n} \leq b_{2}\right\} \cap \Omega\right]
\end{aligned}
$$

Notice that on the set $\Omega$, for $b_{1} \leq B_{n} \leq b_{2}$ we have

$$
\begin{aligned}
\frac{u-Y_{n-1}-\Pi_{n-1} B_{n}}{\Pi_{n-1}\left(Y_{m}^{\prime}-\Pi_{m-1}^{\prime} B_{m}^{\prime}\right)} & <\frac{u}{\gamma u C_{3} / 2}=\frac{2}{\gamma C_{3}}=: C_{+} \\
\frac{u-Y_{n-1}-\Pi_{n-1} B_{n}}{\Pi_{n-1} Y_{m}^{\prime}} & >\frac{u-C_{1} \gamma u-\gamma u b_{1}}{D_{1} \gamma u C_{2}}=\frac{1-C_{1} \gamma-\gamma b_{1}}{D_{1} \gamma C_{2}}=: C_{-} .
\end{aligned}
$$

Here we have used $B_{m}^{\prime} \Pi_{m-1}^{\prime} \leq D_{2} b_{2} \varepsilon \leq \frac{C_{3}}{2}$ for large $m$. Moreover on $\Omega$

$$
\begin{aligned}
\frac{u-Y_{n-1}-\Pi_{n-1} B_{n}}{\Pi_{n-1}\left(Y_{m}^{\prime}-\Pi_{m-1}^{\prime} B_{m}^{\prime}\right)}-\frac{u-Y_{n-1}-\Pi_{n-1} B_{n}}{\Pi_{n-1} Y_{m}^{\prime}} \\
\geq \frac{u-Y_{n-1}-\Pi_{n-1} B_{n}}{\Pi_{n-1}} \cdot \frac{\Pi_{m-1}^{\prime} B_{m}^{\prime}}{Y_{m}^{\prime}\left(Y_{m}^{\prime}-\Pi_{m-1}^{\prime} B_{m}^{\prime}\right)} \\
\geq \frac{1-C_{1} \gamma-\gamma b_{1}}{D_{1} \gamma} \cdot \frac{\varepsilon b_{1}}{C_{2}^{2}}=d_{1} \varepsilon .
\end{aligned}
$$

and we have $C_{-}>0, d_{1}>0$ if we take $\left(C_{1}+b_{1}\right) \gamma \leq \frac{1}{2}$.

The set $\Omega$ is independent on $A_{n}, B_{n}$. By (6.6) for fixed value of $b=B_{n}$, we can take any $A_{n}$ in (6.6) from some interval $I_{d_{1} \varepsilon, b} \subset\left(C_{-}, C_{+}\right)$(depending on $b$ ) of length at least $d_{1} \varepsilon$. In view of $1.17 d_{2}=\inf _{C_{-}<a<C_{+}} g_{A}(a)$ is strictly positive. Then, by (6.5)

$$
\mathbb{P}\left[Y_{k_{u}-1}<u, Y_{k_{u}}>u\right] \geq \inf _{I_{d_{1} \varepsilon, b} \subset\left(C_{-}, C_{+}\right)} \mathbb{P}\left[A \in I_{d_{1} \varepsilon}\right] \mathbb{P}[\Omega] \geq C d_{1} d_{2} \cdot \frac{\lambda(\beta)^{n}}{u^{\beta}} \frac{1}{\sqrt{n}} \frac{\lambda(1)^{m}}{\sqrt{m}}
$$

Since $\sqrt{n m}$ is of order $\log u$, to finish the proof we have to justify that

$$
\frac{\lambda(\beta)^{n} \lambda(1)^{m}}{u^{\beta}} \geq \frac{\lambda(\alpha)^{k_{u}}}{u^{\alpha}} \cdot u^{\delta}
$$

for some $\delta$. In other words we want to show

$$
\left(\frac{\lambda(\beta)}{\lambda(\alpha)}\right)^{n}\left(\frac{\lambda(1)}{\lambda(\alpha)}\right)^{m} \geq u^{\beta-\alpha} u^{\delta} .
$$

Choose $p$ in (6.4) such that $\beta-\alpha<\eta$ for $\eta=\frac{\log \mu}{\Lambda^{\prime}(\alpha)}$ and $\mu=\frac{\lambda(1)}{\lambda(\alpha)}>1$. Then, by the Taylor expansion of $\Lambda$, since $\Lambda^{\prime \prime}(\beta)>0, \Lambda(\beta)-\Lambda(\alpha) \geq \Lambda^{\prime}(\alpha)(\beta-\alpha)$ and so

$$
\begin{aligned}
\left(\frac{\lambda(\beta)}{\lambda(\alpha)}\right)^{n}\left(\frac{\lambda(1)}{\lambda(\alpha)}\right)^{m} & =e^{n(\Lambda(\beta)-\Lambda(\alpha))} \mu^{m} \\
& \geq e^{n(\beta-\alpha) \Lambda^{\prime}(\alpha)} \mu^{m}
\end{aligned}
$$


But $n(\beta-\alpha) \Lambda^{\prime}(\alpha)=(\beta-\alpha) p \log u$ and $\mu^{m}=e^{\log \mu \cdot \frac{q \log u}{\Lambda^{\prime}(\alpha)}}=e^{q \eta \log \mu}$. Hence

$$
\begin{aligned}
\left(\frac{\lambda(\beta)}{\lambda(\alpha)}\right)^{n}\left(\frac{\lambda(1)}{\lambda(\alpha)}\right)^{m} & \geq u^{p(\beta-\alpha)} u^{\eta q} \\
& =u^{\beta-\alpha} u^{(\eta-(\beta-\alpha)) q}
\end{aligned}
$$

which completes proof of the Theorem.

Remark 6.7. The assumption on $g_{A}(a)$ in (1.17) can be weakened to $g_{A}(a) \geq c>0$ on some interval $\left(a_{1}, a_{2}\right)$ with $a_{1}>1$. The proof is similar. It requires only a more careful definition of $\Omega$. If $g_{A}(a) d a$ contains a nontrivial absolutely continous part, by Steinhaus theorem, its convolution power has to satisfy the condition above. We leave the details for the reader.

\section{REFERENCES}

[1] D. Buraczewski. On tails of fixed points of the smoothing transform in the boundary case. Stochastic Process. Appl. 119, 3955-3961, 2009.

[2] D. Buraczewsi, J. Collamore, E. Damek, and J. Zienkiewicz. Large deviation estimates for exceedance times of perpetuity sequences and their dual processes. Ann. Probab., to appear.

[3] D. Buraczewski, E. DameK. A simple proof of heavy tail estimates for affine type Lipschitz recursions. Stochastic Process. Appl. 127, 657-668, 2017.

[4] D. Buraczewski, E. Damek, T. Mikosch and J. Zienkiewicz. Large deviations for solutions to stochastic recurrence equations under Kesten's condition. Ann. Probab., Vol. 41, No. 4, 2755-2790, 2013.

[5] D. Buraczewski, E. Damek And T. Mikosch. Stochastic models with power-law tails: the equation $X=A X+B$. Springer, 2016 .

[6] D. Buraczewski, E. Damek and J. Zienkiewicz. Precise tail asymptotics of fixed points of the smoothing transform with general weights Bernoulli, Vol. 21(1), 489-504, 2015.

[7] D. Buraczewski, M. Maślanka. Precise large deviations for the first passage time of random walk with negative drift Proc. AMS, to appear.

[8] J. F. Collamore and A. N. Vidyashankar. Tail estimates for stochastic fixed point equations via nonlinear renewal theory. Stochastic Process. Appl. 123, 3378-3429, 2013.

[9] A. Dembo and O. Zeitouni. Large Deviations Techniques and Applications. Springer 1998.

[10] R. F. Engle. Autoregressive conditional heteroscedasticity with estimates of the variance of United Kingdom inflation. Econometrica 50, 987-1007, 1982.

[11] N. Enriquez, C. Sabot and O. Zindy. A probabilistic representation of constants in Kesten's renewal theorem. Probab. Theory Related Fields 144, no. 3-4, 581-613, 2009.

[12] C. M. Goldie. Implicit renewal theory and tails of solutions of random equations. Ann. Appl. Probab. 1, 1, 126-166, 1991.

[13] Y. Guivarc'H. Sur une extension de la notion de loi semi-stable. Ann. Inst. H. Poincaré Probab. Statist. 26, 261-285, 1990.

[14] A. Iksanov. Renewal theory for perturbed random walks and similar processes. Birkhäuser, 2016.

[15] H. Kesten. Random difference equations and renewal theory for products of random matrices. Acta Math. 131, 207-248, 1973.

[16] H. Kesten, M. Kozlov And F. Spitzer A limit law for random walk in a random environment Compositio Mathematica 30, 145-168, 1975.

[17] S. Lalley. Limit theorems for first-passage times in linear and nonlinear renewal theory. Adv. in Appl. Probab. 16, no. 4, 766-803., 1984

[18] Q. Liu. On generalised multiplicative cascades. Stochastic Process. Appl. 86, 263-286, 2000. 
[19] T. Mikosch. Modeling dependence and tails of financial time series. In B. Finkenstädt and H. Rootzén (Eds.), Extreme Values in Finance, Telecommunications, and the Environment, pp. 185-286. Boca Raton: Chapman and Hall., 2003

[20] H. Nyrhinen. Finite and infinite time ruin probabilities in a stochastic economic environment. Stochastic Process. Appl. 92 (2), 265-285, 2001.

[21] H. Nyrhinen. On the ruin probabilities in a general economic environment. Stochastic Process. Appl. 83 (2), 319-330, 1999.

[22] V. Petrov. On the probabilities of large deviations for sums of independent random variables. Theory Probab. Appl. 10, 287-298, 1965.

[23] V. Petrov. Limit Theorems of Probability Theory, Volume 4 of Oxford Studies in Probability, 1995.

D. Buraczewski, E. Damek, and J. Zienkiewicz, Instytut Matematyczny, Uniwersytet WroClawski, 50-384 Wroclaw, Pl. Grunwaldzki 2/4, Poland

E-mail address: dbura@math.uni.wroc.pl, edamek@math.uni.wroc.pl, zenek@math.uni.wroc.pl 